\title{
Traditionelle Herrscher in moderner Gefahr
}

\author{
Soldatisch-aristokratische Tugendhaftigkeit und das \\ Konzept der Sicherheit im späten 19. Jahrhundert*
}

von Carola Dietze und Frithjof Benjamin Schenk

\begin{abstract}
Contributing to the current debate on the history of security, this essay examines the value "security" had in the late 19th century as a normative maxim for European rulers. It gives examples of attacks on state and government leaders and analyzes their reactions and the resultant discussions about the protection of monarchs. These case studies will demonstrate that the security culture of the heads of European states was shaped more by the traditional model of aristocratic, military and manly virtuousness than by security considerations well into the late 19 th century. Yet the evolution of modern terrorism soon revealed the limits of this traditional security culture.
\end{abstract}

\section{Einleitung: Das Attentat auf Alexander II. im März 1881 und die Frage der Sicherheit von Herrschern}

Nach dem russischen Kalender war der 1. März 1881 ein Sonntag. Am Morgen dieses Tages besprach Zar Alexander II. mit dem Vorsitzenden des Ministerkomitees Petr Aleksandrovič Valuev die Frage, in welcher Form die liberalen Neuerungen der Staatsverfassung, die der Kaiser einige Tage zuvor gebilligt hatte, der Öffentlichkeit bekannt gegeben werden sollten. Nach Abschluss der Gespräche fuhr er mit der Kutsche zum Petersburger Ingenieurpalast und nahm dort die Sonntagvormittagsparade ab. Dies geschah gegen den ausdrücklichen Rat seines Innenministers Michail Loris-Melikov. Denn nur zwei Tage zuvor war Andrej Željabov, ein Führer der Terroristenorganisation Narodnaja volja (Volksfreiheit oder auch Volkswille) verhaftet worden und hatte bei seiner Festnahme prophezeit, die Attentatsversuche gingen auch ohne ihn

* Eine erste Version der Teile I-III und V hat Carola Dietze im Sommer 2007 während der Summer School des Berliner Kollegs für Vergleichende Geschichte Europas an der FU Berlin vorgestellt. Für Diskussion und Anregungen dankt sie allen Teilnehmern der Summer School und insbesondere spiritus rector und Organisatorin Tatjana Tönsmeyer sowie Friedrich Lenger. Frithjof Benjamin Schenk hat frühere Versionen des vierten Teils bei verschiedenen Anlässen in Deutschland und den USA vorgetragen. Für Anregungen, Diskussion und Kritik der Aufsatzfassung danken beide Autoren Martin Aust, Reinhard Frötschner, Anke Hilbrenner, Friedrich Lenger, Jan Plamper, Julija Safronova, Henning Trüper und Claudia Verhoeven.

Geschichte und Gesellschaft 35. 2009, S. 368 - 401

(c) Vandenhoeck \& Ruprecht GmbH \& Co. KG, Göttingen 2009

ISSN 0340-613X 
weiter. Wegen der angespannten Sicherheitslage hatte der Zar bereits an den beiden vorausgegangenen Wochenenden auf die Abnahme der sonntäglichen Parade verzichtet. Doch am 1. März 1881 vertraute er wieder den Sicherheitsmaßnahmen: Für den Herrscher über ganz Russland stand eine gepanzerte Kutsche bereit, eine Spezialanfertigung aus Paris, die Napoleon III. kurz vor seinem Sturz in Auftrag gegeben hatte. Sie sollte den Zaren auf stets wechselnden Routen, die immer erst im letzten Moment bekannt gegeben wurden, ans Ziel bringen. Die entsprechenden Straßen wurden jeweils für die Öffentlichkeit gesperrt und unterstanden besonderer Bewachung. Nach dem Ende der Sonntagsvormittagsparade begab sich Alexander II. auf den Weg zurück zum Winterpalast, ließ unterwegs aber am Michajlovskij-Palast halten, wo er seiner Cousine, der Großfürstin Katharina, einen kurzen Besuch abstattete. Für den weiteren Weg zum Palast befahl er seinem Kutscher, als Abkürzung die Katharinenstraße zu nehmen, eine ruhige Seitenstraße, die nicht für die Öffentlichkeit gesperrt worden war. So konnte der neunzehnjährige Bergbaustudent Nikolaj Rysakov um 13.45 Uhr in der Nähe der Theaterbrücke eine Bombe auf die kaiserliche Kutsche werfen. Die Spezialanfertigung aus Paris hielt der Detonation jedoch stand, allein einige Kosaken aus der Eskorte wurden verletzt. Allerdings musste Alexander II. die Kutsche nun verlassen. Wäre es nach dem Rat seiner Eskorte gegangen, wäre er schnell in den Schlitten umgestiegen, der die Kutsche begleitete. Doch der Imperator bestand darauf, den Verletzten zu helfen. In dem, was daraufhin geschah, unterscheiden sich die Berichte. Einer Beschreibung zufolge soll Alexander II. auf die Frage Umstehender, ob er selbst verletzt sei, geantwortet haben, er sei gottlob unversehrt. Darauf soll Rysakov bemerkt haben, es sei zu früh, Gott zu danken, woraufhin Ignatij Grineveckij, ein Student polnischer Herkunft vom Technologischen Institut, seine Bombe Alexander II. direkt vor die Füße warf. Fest steht, dass diese zweite Detonation Alexander II. beide Beine bis unter die Knie zerschmetterte, den Unterleib aufriss und die rechte Hand zerfetzte. Bewusstlos wurde er mit dem Schlitten zum Winterpalast gebracht, wo er trotz Notamputation um 15.35 Uhr verstarb. ${ }^{1}$

1 Für zeitgenössische Schilderungen des Attentats vgl. Viktor Efimovič Kel'ner (Hg.), 1 marta 1881 goda. Kazn' imperatora Aleksandra II. Dokumenty i vospominanija, Leningrad 1991. Die klassische Darstellung des Ereignisses in der historischen Literatur findet sich bei Sergej Spiridonovič Tatiščev, Imperator Aleksandr Vtoroj. Ego žizn’ i carstvovanie [1902], Moskau 2006, S. 947 f. Für eine neuere Beschreibung siehe etwa Evgenij Petrovič Tolmačev, Aleksandr II i ego vremja. Kniga vtoraja, Moskau 1998, S. 229 f. oder Hans-Joachim Torke, Die Narodniki und Zar Alexander II. Ein Vorspiel zur Revolution [1881], in: Alexander Demandt (Hg.), Das Attentat in der Geschichte, Köln 1996, S. 251 -265. Zur öffentlichen Reaktion auf das Ereignis vgl. Julija Safronova, Smert' gosudarja. 1 marta 1881 goda. Ėmocional'nyj srez, in: Jan Plamper u. a. (Hg.), Rossijskaja imperija čuvstv. Podchody k kul'turnoj istorii èmocij. Moskau [2010]. 
Anstatt so schnell wie möglich den Ort der Gefahr zu verlassen, kümmerte sich Alexander II. um die verletzten Leibgardisten und gab damit den Terroristen eine zweite Chance, ihn zu töten. Wie ist das unvorsichtige Verhalten des Alleinherrschers über ganz Russland in dieser Gefahrensituation zu deuten? Verstand er nicht, dass hier eine Verschwörergruppe nach seinem Leben trachtete? Wusste er nicht, wie man sich angesichts einer solchen Bedrohung zu verhalten hatte? Oder gab es andere Vorstellungen, die sein Handeln leiteten?

In seinem programmatischen Aufsatz „Sicherheit als Kultur“ hat Eckart Conze kürzlich auf den heuristischen Wert der Analysekategorie „Sicherheit" für eine neue Politikgeschichte hingewiesen. ${ }^{2}$ Conze, der sich der Geschichte von Sicherheitsvorstellungen am Beispiel der Bundesrepublik Deutschland widmet, betont hier die Historizität von Sicherheitsbewusstsein und Sicherheitsverständnis. Er lenkt den Blick auf die Varianz von Sicherheitsbegriffen und das veränderliche Verständnis von Sicherheit in unterschiedlichen politischen und sozialen Kontexten. Seine Fallstudie verknüpft Conze mit der Forderung nach diachronen Studien, die „Erkenntnisgewinn versprechen über Prozesse sozialen Wandels, der mit historisch-gesellschaftlichen Sicherheitsbedürfnissen und dem damit korrespondierenden Sicherheitsbewusstsein eng verschränkt ist. “3 Sicherheit wird von Conze als „umfassender sozialkultureller Orientierungshorizont" verstanden, der zum einen menschliche Grundbedürfnisse und gesellschaftliche Zukunftsvorstellungen widerspiegle, zum anderen aber auch das Versprechen moderner Staaten auf ein Leben ihrer Bürger in „Gewissheit, Verlässlichkeit, Ruhe und Geborgenheit" reflektiere. Durch diese Doppelperspektive auf gesellschaftliche Wunschvorstellung und politisches Versprechen helfe der Begriff Sicherheit in analytischer Hinsicht, Distanz zwischen „Politik“ und „Gesellschaft“ zu verringern und so ein wichtiges Postulat einer „modernen Politikgeschichte“ zu erfüllen. ${ }^{4}$

2 Eckart Conze, Sicherheit als Kultur. Überlegungen zu einer „modernen Politikgeschichte" der Bundesrepublik Deutschland, in: VfZ 53. 2005, S. 352 -380. Zur Umsetzung dieses Programms vgl. jetzt ders., Die Suche nach Sicherheit. Eine Geschichte der Bundesrepublik Deutschland von 1949 bis in die Gegenwart, München 2009.

3 Conze, Sicherheit als Kultur, S. $360 \mathrm{f}$.

4 Ebd., S. 360, S. 365 u. S. 359. Zur Debatte um die „moderne Politikgeschichte“ vgl. einführend ders., „Moderne Politikgeschichte“. Aporien einer Kontroverse, in: Guido Müller (Hg.), Deutschland und der Westen. Festschrift für Klaus Schwabe zum 65. Geburtstag (= Historische Mitteilungen, Bd. 29), Stuttgart 1998, S. 19-30. Zu Sicherheit und Sicherheitspolitik im Allgemeinen sowie zu den verschiedenen Bereichen von Sicherheitspolitik (etwa der äußeren, inneren, sozialen Sicherheit) gibt es eine umfangreiche Spezialliteratur. Eine gute Einführung in die historische Forschung wie die aktuelle Diskussion geben Martin Dinges u. Fritz Sack, Unsichere Großstädte?, in: Martin Dinges u. Fritz Sack (Hg.), Unsichere Großstädte? Vom Mittelalter bis zur Postmoderne (= Konflikte und Kultur. Historische Perspektiven, 
Die folgenden Überlegungen knüpfen an die aktuelle Debatte über die Geschichte von Sicherheitsbewusstsein und Sicherheitsbedürfnissen in der neueren und neuesten europäischen Geschichte an. Sicherheit wird hier jedoch nicht in ihrer ganzen Breite als „sozialkultureller Orientierungshorizont“ im gesellschaftlichen Diskurs gefasst, sondern es wird konkreter danach gefragt, wann und unter welchen Bedingungen sich Sicherheit zu einer Kategorie entwickelte, die für das Verhalten von europäischen Staatsoberhäuptern handlungsleitend war. Am Beispiel einiger Attentate auf Staats- und Regierungsoberhäupter, deren Reaktionen sowie der Diskussion um den Schutz der körperlichen Unversehrtheit von Monarchen soll gezeigt werden, dass das Handeln europäischer Herrscher im späten 19. Jahrhundert noch nicht in dem Maße von der Kategorie Sicherheit bestimmt wurde, wie wir es vom Verhalten politischer Führungsfiguren und den zu ihrem Schutz bestimmten Institutionen aus dem 20. oder beginnenden 21. Jahrhundert kennen. Zwar zählte der Schutz des Lebens von Regenten traditionell zu den Grundprinzipien politischer Herrschaftssicherung. Doch war die Sicherheitskultur gerade der Spitzen der europäischen Staaten bis ins späte 19. Jahrhundert noch stark von traditionellen Verhaltensnormen geprägt.

$\mathrm{Zu}$ diesen traditionellen Vorstellungen und Handlungsmustern zählten das Leitbild soldatisch-aristokratischer Tugendhaftigkeit und die Überzeugung vom göttlichen Schutz des durch höchste Gnaden legitimierten Herrschers. Die soldatisch-aristokratischen Tugenden waren über Mut und Todesverachtung sowie Ehre und Haltung definiert: Es galt, Gefahren ins Auge zu blicken und Gegnern die Stirn zu bieten. Dabei waren Monarchen den Anforderungen dieser Tugenden als erste Offiziere ihrer Nationen in besonderem Maße unterworfen. Wenn sie von ihren Offizieren in Verordnungen und Gutachten zum Offizierskorps Geradlinigkeit, Entschlusskraft und Mut verlangten sowie „standhafte Unerschrockenheit in jeder Gefahr“, die sich in einer Situation der Bedrohung bis zur Lebensverachtung steigern müsse, so hatten sie diese Tugenden zuallererst selbst auf vorbildliche Weise zu verkörpern. Hinzu kamen die Ansprüche, die das Ideal der Ritterlichkeit an das Verhalten des adligen Mannes der Hocharistokratie stellte: es ging darum, Haltung zu zeigen - und zwar als Affektbeherrschung und Körperkontrolle - sowie Höflichkeit und Ehrenhaftigkeit unter Beweis zu stellen und dadurch auch der eigenen her-

Bd. 3), Konstanz 2000, S. 9-65. Zur Geschichte des Begriffs vgl. nach wie vor Werner Conze, Sicherheit, Schutz, in: Otto Brunner u. a. (Hg.), Geschichtliche Grundbegriffe. Historisches Lexikon zur politisch-sozialen Sprache in Deutschland, Bd. 5, Stuttgart 1984, S. 831-862. Zur Veränderung von Sicherheit und Sicherheitsbedürfnissen in der Moderne aus soziologischer Sicht vgl. Wolfgang Sofsky, Das Prinzip Sicherheit, Frankfurt 2005. Für eine philosophische Perspektive vgl. Hermann Lübbe, Die schwarze Wand der Zukunft, in: Ernst Peter Fischer (Hg.), Auf der Suche nach der verlorenen Sicherheit, München 1991, S. 17-31. 
ausragenden sozialen Stellung Ausdruck zu verleihen. ${ }^{5}$ Diese Leitvorstellungen wurden am Ende des 19. Jahrhunderts in Europa von einem großen Teil der Gesellschaft geteilt. Sie spiegelten damit auch eine Erwartungshaltung wider, wie sich der regierende Fürst in einer für sein Leben gefährlichen Situation zu verhalten habe. Der Selbsterhalt, das pure Überleben waren in dieser Vorstellungswelt nicht das allerhöchste Gut, sondern dem Begriff der Ehre - der persönlichen wie jener der Dynastie oder gar der ganzen Nation - untergeordnet. ${ }^{6}$ So wurden die Überwindung von Gefahr oder das Meistern einer gefährlichen Situation als Beweis von Standhaftigkeit und Stärke sowie als Ausdruck göttlicher Gnade interpretiert und in ein Argument zur Steigerung der Legitimität politischer Herrschaft verwandelt, während das Ausweichen oder der Rückzug zum Selbstschutz als Zeichen von Schwäche galten. Von

$5 \mathrm{Zu}$ den Anforderungen an die Offiziersehre, die etwa von Wilhelm I. für das preußische Heer festgeschrieben wurden, vgl. Ute Frevert, Ehrenmänner. Das Duell in der bürgerlichen Gesellschaft, München 1991, Kap. 4. Das Zitat findet sich auf S. 107. Zu den Anforderungen an den adligen Mann vgl. für Preußen ebd. S. 100 f. sowie dies., Das Militär als „Schule der Männlichkeit“. Erwartungen, Angebote, Erfahrungen im 19. Jahrhundert, in: dies. (Hg.), Militär und Gesellschaft im 19. und 20. Jahrhundert (= Industrielle Welt. Schriftenreihe des Arbeitskreises für moderne Sozialgeschichte, Bd. 58), Stuttgart 1997, S. 145 - 173 und Marcus Funck, Vom Höfling zum soldatischen Mann. Varianten und Umwandlungen adeliger Männlichkeit zwischen Kaiserreich und Nationalsozialismus, in: Eckart Conze u. Monika Wienfort (Hg.), Adel und Moderne. Deutschland im europäischen Vergleich im 19. und 20. Jahrhundert, Köln 2004, S. 205 -235. Die Attribute lassen sich vermutlich auf das am preußischen Vorbild orientierte russische Militär und den russischen Adel übertragen. Vgl. zum russischen Adel einführend Manfred Hildermeier, Der russische Adel von 1700 bis 1917, in: Hans-Ulrich Wehler (Hg.), Europäischer Adel 1750 - 1950 (= GG Sonderheft 13), Göttingen 1990 und zur Rolle des Adels im russischen Militär Dietrich Beyrau, Militär und Gesellschaft im vorrevolutionären Russland (= Beiträge zur Geschichte Osteuropas, Bd. 15), Köln 1984.

6 Die Bedeutung solcher aristokratischen Habitus- und Distinktionsformen auch in der bürgerlichen Gesellschaft des 19. Jahrhunderts sind seit Ende der achtziger Jahre von einer kulturgeschichtlich sensiblen politischen Sozialgeschichte verstärkt herausgearbeitet und untersucht worden. Vgl. einführend Heinz Reif, Adel im 19. und 20. Jahrhundert (= Enzyklopädie deutscher Geschichte, Bd. 55), München 1999 sowie vor allem die europäisch vergleichenden Bände Les noblesses européennes au XIXe siècle hg. v. École française de Rome / Centro per gli studi di politica esterna e opinione pubblica de l'Université de Milan (= Collection de l'école francaise de Rome, Bd. 107), Paris 1988; Armgard von Reden-Dohna u. Ralph Melville, Der Adel an der Schwelle des bürgerlichen Zeitalters 1780-1860 (=Veröffentlichungen des Instituts für europäische Geschichte Mainz, Abteilung Universalgeschichte 10), Stuttgart 1988; Hans-Ulrich Wehler (Hg.), Europäischer Adel 1750-1950, Göttingen 1990 (= GG Sonderheft 13) und Eckart Conze, Adel und Moderne. Deutschland im europäischen Vergleich im 19. und 20. Jahrhundert, Köln 2004. 
einem solchen Standpunkt aus betrachtet, erscheint das Verhalten Alexanders II., der am 1. März 1881 nicht die Flucht vor seinen Attentätern ergriff, folgerichtig, ja sogar vorbildlich und beinahe zwingend.

Der gewaltsame Tod des Reformzaren am Petersburger Katharinenkanal verdeutlicht jedoch in gleichem Maße, wie unzeitgemäß die Orientierung der europäischen Monarchen an soldatisch-aristokratischen Tugenden im späten 19. Jahrhundert geworden war. Alexander II. wurde nicht zuletzt zum Opfer einer für das autokratische Regime zunehmend gefährlichen Gleichzeitigkeit des Ungleichzeitigen. ${ }^{7}$ Die Mitglieder der Terrororganisation Narodnaja volja waren in Bezug auf ihre Angriffsmethoden und die Wahl ihrer Waffen sehr viel fortschrittlicher - und das heißt, schon deutlicher im Industrie- und Medienzeitalter angekommen - als der Zar in seinem Umgang mit den von ihnen ausgehenden Gefahren. Insofern läutete nicht zuletzt der moderne Terrorismus mit seiner Offenheit für den technischen Fortschritt den langsamen Abschied von soldatisch-aristokratischen Handlungsmaximen europäischer Staatsoberhäupter sowie eine Technisierung und Entpersonalisierung der Maßnahmen zu deren persönlichem Schutz ein. Der Abschied von der Kardinaltugend des Mutes, der für die Geschichte der Sicherheit festgestellt werden kann, lässt sich dabei mit dem Abschied von der Ritterlichkeit des Kampfes im Zuge der zunehmenden Industrialisierung des Krieges vergleichen, den die Militärgeschichte beschreibt. ${ }^{8}$

Im Mittelpunkt der folgenden Betrachtungen steht die Frage, welchen Stellenwert die Kategorie der Sicherheit in der zweiten Hälfte des 19. Jahrhunderts als handlungsleitende Maxime für europäische Staats- und Regierungsoberhäupter entwickeln konnte. Im Vergleich mit ausgewählten Beispielen aus verschiedenen europäischen Ländern wird der Wandel von Handlungsmus-

$7 \mathrm{Zu}$ dem von Wilhelm Pinder und Ernst Bloch geprägten Konzept der „Gleichzeitigkeit des Ungleichzeitigen " und seiner Geschichte vgl. Siegfried Kracauer, History. The Last Things Before the Last. Completed after the Death of the Author by Paul Oskar Kristeller [1969], Princeton 1995, Kap. 6 sowie Frederic J. Schwartz, Ernst Bloch and Wilhelm Pinder. Out of Sync, in: Grey Room 3. 2001, S. 54-89. Vor allem Reinhart Koselleck hat die Denkfigur in die Geschichtswissenschaft eingeführt. Vgl. zum Beispiel Reinhart Koselleck, Die agrarische Grundverfassung Europas zu Beginn der Industrialisierung, in: Louis Bergeron u.a. (Hg.), Das Zeitalter der europäischen Revolution 1780 - 1848, Frankfurt 1969, S. $230-261$.

8 Vgl. dazu abstrakt für die Geschichte der Sicherheit Sofsky, Das Prinzip Sicherheit, insbesondere Kap. 4. Für die Militärgeschichte vgl. einführend Edgar Wolfrum, Krieg und Frieden in der Neuzeit. Vom Westfälischen Frieden bis zum Zweiten Weltkrieg, Darmstadt 2003, S. 105-107 sowie den eindrücklichen und anschaulichen Band von Rolf Spilker u. Bernd Ulrich (Hg.), Der Tod als Maschinist. Der industrialisierte Krieg 1914/1918. Eine Ausstellung des Museums Industriekultur Osnabrück im Rahmen des Jubiläums „350 Jahre Westfälischer Friede“, 17.5.-23.8.1998, Bramsche 1998, und hier vor allem die Aufsätze von Klaus Latzel und Benjamin Ziemann. 
tern europäischer Regenten am russischen Fall exemplarisch untersucht. Damit soll nicht zuletzt der Rahmen für eine komparative Analyse der Sicherheitsvorstellungen von Staatsoberhäuptern und Regierungen im Europa des späten 19. Jahrhunderts abgesteckt werden.

\section{Ein Beispiel an soldatisch-aristokratischer Tugendhaftigkeit: Das Verhalten Zar Alexanders II. gegenüber seinen Attentätern}

Wenige im 19. Jahrhundert verübte Herrscherattentate sind so prominent und auch heute noch so fest im kollektiven Gedächtnis innerhalb und außerhalb Russlands verankert wie das Attentat auf Zar Alexander II. vom 1. März 1881. Ein Grund dafür ist in der historischen Bedeutung dieses politischen Mordes zu sehen. Denn sein Nachfolger, Alexander III., setzte die vorsichtige Liberalisierungspolitik seines Vaters nicht fort. Russland blieb bis in das 20. Jahrhundert hinein eine Autokratie, die breiten Kreisen der Bevölkerung kaum politische Gestaltungsmöglichkeiten bot und wenig Rückhalt im städtischen Bürgertum und bei der Intelligenz genoss. Die mittelbaren Folgen dieser restaurativen Politik für die Geschichte des 20. Jahrhunderts sind bekannt.

Ein weiterer Grund für die herausragende Stellung des Attentats von $1881 \mathrm{im}$ europäischen Geschichtsbewusstsein ist die Tatsache, dass der Anschlag den Schlusspunkt einer langen Serie spektakulärer terroristischer Angriffe auf den Imperator markierte, die Russland in der Wahrnehmung vieler Zeitgenossen und zahlreicher Historiker gar als das „Mutterland des modernen Terrorismus" erscheinen lassen. ${ }^{9}$ Bereits im Jahre 1866 hatte Dmitrij Karakosov, ein Student, der von der Universität Kazan' verwiesen worden war, den ersten Mordanschlag auf den Alleinherrscher über ganz Russland versucht. Ein Jahr später schoss der Pole Alexander Berezowski auf den Zaren, als dieser in Paris weilte, um sich die Weltausstellung anzusehen. Und 1879 gab der Volkstümler (narodnik) Aleksandr Solov'ev fünf Schüsse auf den Kaiser ab, die jedoch alle ihr Ziel verfehlten. Dieser Attentatsversuch war innerhalb des revolutionären Lagers noch heftig umstritten gewesen. Nach diesem Fehlschlag konnte sich der radikale Flügel der Volkstümler jedoch gegen interne Zweifler durchsetzen und - nach der Gründung der Geheimorganisation Narodnaja volja im Sommer 1879 - ein „Todesurteil“ über Alexander II. verhängen. Dies geschah nicht zuletzt deshalb, weil die Revolutionäre befürchteten, die missglückten Attentate könnten die russischen Bauern womöglich in ihrem Glauben bestärken,

9 Vgl. z. B. Walter Laqueur, A History of Terrorism, New Brunswick 2002², S. 31; Gérard Chaliand u. Arnaud Blin, The History of Terrorism from Antiquity to Al Quaeda, Berkeley 2007, S. 111 sowie Anna Geifman, Thou Shalt Kill. Revolutionary Terrorism in Russia, 1894 - 1917, Princeton 1993. 
der Zar stehe unter Gottes Schutz. Es galt, das Gegenteil zu beweisen. In den darauf folgenden achtzehn Monaten unternahm die Organisation noch ein halbes Dutzend weitere Attentatsversuche, darunter zwei Bombenanschläge auf den kaiserlichen Zug, in dem der Zar von der Krim nach Petersburg zurückreiste, und - besonders spektakulär - ein Bombenattentat im Winterpalast. $^{10}$

Doch obgleich Alexander II. einer solchen Hetzjagd ausgesetzt war, veränderte er sein Verhalten in einem entscheidenden Punkt nicht: Er ging auf seine Attentäter zu und sprach sie an. Bei dem ersten Attentat Karakosovs mag man dies noch mit der Unerfahrenheit des Imperators erklären, wie man sich in einer solchen Situation zu verhalten habe. An jenem 4. April des Jahres 1866 war er wie beinahe jeden Nachmittag um diese Zeit im Sommergarten spazieren gegangen. Wie gewöhnlich blieb der Park während der Anwesenheit des Zaren für die Öffentlichkeit zugänglich. Zwar hatten die zwei Polizisten, die den Eingang zum Park bewachten, Instruktionen, Personen den Zutritt zu verwehren, die schlecht gekleidet waren oder sich auffällig verhielten. Aber es wäre für jeden ein leichtes gewesen, unbemerkt über den schmiedeeisernen Zaun zu klettern, der den Sommergarten umgibt. Als der Zar von seinem Spaziergang zurückkam, eilten die beiden Polizisten, dem Kaiser den Mantel abzunehmen und ihm die Tür zur Kutsche zu öffnen, die vor dem Tor des Sommergartens auf ihn wartete. Auf die Zuschauer, die sich wie üblich in respektvollem Abstand rings um die Kutsche versammelt hatten, achteten sie nicht. So bemerkten sie auch nicht, dass einer der Umstehenden eine Pistole zog, sie auf den Zaren richtete und abdrückte. Der Schuss traf nicht, weil angeblich der Bauernsohn Osip Komissarov den Arm, mit dem Karakosov die Pistole hielt, im rechten Moment zur Seite stieß. Zwar hatte Karakosov noch einen zweiten Schuss in seiner Pistole, doch versuchte er jetzt zu fliehen. Während alle Umstehenden vor Schreck wie erstarrt waren, liefen die beiden

10 Zur Geschichte der revolutionären Bewegungen in Russland bis 1881 und ihren Attentaten vgl. einführend Astrid von Borcke, Gewalt und Terror im revolutionären Narodničestvo. Die Partei „Narodnaja volja“, 1879-1883, in: Wolfgang J. Mommsen u. Gerhard Hirschfeld (Hg.), Sozialprotest, Gewalt, Terror. Gewaltanwendung durch politische und gesellschaftliche Randgruppen im 19. und 20. Jahrhundert (= Veröffentlichungen des Deutschen Historischen Instituts London, Bd. 10), Stuttgart 1982, S. 64 - 79 sowie die klassischen Darstellungen von Franco Venturi, Roots of Revolution. A History of the Populist and Socialist Movements in 19th Century Russia [1952], London 2001; Philip Pomper, The Russian Revolutionary Intelligentsia, New York 1970; Adam Bruno Ulam, In the Name of the People. Prophets and Conspirators in Prerevolutionary Russia [1977], New Brunswick 1998; Norman M. Naimark, Terrorism and the Fall of Imperial Russia, in: Terrorism and Political Violence 2. 1990, S. 171 - 192 und die entsprechenden Kapitel in Oleg Vital'evič Budnickij, Terrorizm v rossijskom osvoboditel'nom dviženii. Ideologija, ètika, psichologija (vtoraja polovina XIX - načalo XX v.), Moskau 2000. 
Polizisten dem Attentäter nach und holten ihn ein. Sie hielten Karakosov fest und nahmen ihm die Pistole ab. Doch anstatt dass Alexander II. nun schnell in die Kutsche gestiegen und zum Palast zurückgefahren wäre - schließlich hätte unter den Zuschauern noch ein Komplize sein oder Karakosov noch eine weitere Waffe bei sich tragen können - ging der Imperator auf seinen Untertan $\mathrm{zu}$ und fragte ihn, wer er sei und was er wolle. "Ein Russe“, erhielt er zur Antwort und: „Nichts, nichts. “ Der Attentäter wurde von den beiden Polizisten abgeführt. Eine Menschenmenge folgte ihnen, bis die Polizisten eine Droschke anhielten und den Verbrecher auf diesem Wege hinter Schloss und Riegel brachten. ${ }^{11}$

Die Angewohnheit, auf seine Attentäter zuzugehen und sie anzusprechen, behielt Alexander II. bei. Dabei kann man ihm nicht vorwerfen, die Anschläge auf sein Leben auf die leichte Schulter genommen zu haben. Während der nächsten fünfzehn Jahre wurden zahlreiche Beamte und Politiker, die mit dem Schutz der Sicherheit des Monarchen betraut waren, ausgetauscht, Untersuchungskommissionen eingerichtet sowie Gesetze erlassen oder verschärft mit dem Ziel, den sozialradikalen Terrorismus in Russland effektiver zu bekämpfen: So übertrug der Zar die Leitung der Untersuchungskommission für den Fall Karakosov dem Grafen Michail Murav'ev, der sich wegen seiner drakonischen Maßnahmen zur Niederschlagung des polnischen Januaraufstands bereits als „Henker von Polen“ einen Namen gemacht hatte. Murav'ev ordnete an, jeden, der auch nur irgendwie verdächtig sein konnte, festzunehmen und zu verhören. Viele Publizisten und Schriftsteller befanden sich unter den Betroffenen. Der liberale Generalgouverneur St. Petersburgs, Fürst Aleksandr A. Suvorov, sowie der Chef der Geheimpolizei und der Gendarmen, Fürst Vasilij A. Dolgorukov, mussten von ihren Ämtern zurücktreten. Nach dem Attentat Aleksandr Solov'evs im Jahr 1879 wurden die Generalgouverneure für Petersburg, Odessa und Char'kov ausgetauscht und ihre Nachfolger mit Sonderrechten ausgestattet: Sie konnten nun nach Gutdünken Zeitungen verbieten, Zivilpersonen vor Militärgerichte stellen, Verhaftungen vornehmen und Delinquenten nach Sibirien verbannen. Nach dem Bombenanschlag im Winterpalast wurde eine Kommission mit unbegrenzten Befugnissen zur Bekämpfung der Terroristen eingesetzt. All dies belegt, dass der Zar die Attentate und die Gefahren für seine eigene Person durchaus ernst nahm. ${ }^{12}$

11 Zum Attentat Karakosovs vgl. Efim Grigor'evič Zil'berman u. Valentin Konstantinovič Choljavin, Vystrel. Očerk žizni i revoljucionnoj bor'by D. Karakosova, Kazan' 1968; Adam B. Ulam, In the Name of the People, S. 1-8; Nikolaj P. Eroškin, Vystrel u Letnogo Sada, in: Voprosy Istorii, 1993, Nr. 7, S. 170-173 sowie jetzt vor allem Claudia Verhoeven, The Odd Man Karakozov. Imperial Russia, Modernity, and the Birth of Terrorism, Ithaca 2009.

$12 \mathrm{Vgl}$. zu diesen und anderen von Alexander II. und seiner Regierung ergriffenen Maßnahmen die in den Anm. 9 und 10 genannte Literatur. 
Und dennoch ließ sich Alexander II. den unmittelbaren Kontakt mit seinen Untertanen nicht nehmen. ${ }^{13}$ So musste Michail Frolenko, der zum inneren Zirkel der Narodnaja volja gehörte, erstaunt und in stummer Wut mit ansehen, wie der Zar 1879 während eines Zwischenstopps an einem Bahnhof im Gouvernement Orel aus dem kaiserlichen Zug ausstieg, um ein bisschen mit den Bäuerinnen zu schwatzen und zu scherzen, die sich an der Station versammelt hatten und einen Blick auf das Zarenpaar ergattern wollten. ${ }^{14}$ Und nachdem Rysakov am 1. März 1881 eine Bombe auf die kaiserliche Kutsche geworfen hatte, ging Alexander II. auch auf ihn zu und gab auf diese Weise Grineveckij erst die Gelegenheit, seine Bombe einzusetzen. Schon damals stellte der $\mathrm{Pu}$ blizist Stanislas M. Propper fest:

Die Verschworenen hatten auf den Edelmut und die Unerschrockenheit des Kaisers gerechnet. Anstatt weiter zu fahren und schleunigst den gefährdeten Platz zu verlassen, wie es viele Monarchen an seiner Stelle getan hätten, ließ der Kaiser die Pferde halten und stieg aus dem Wagen, um den Verwundeten beizustehen und sie in seinem Wagen unterzubringen. Darauf hatten die Attentäter gebaut. ${ }^{15}$

\section{Zar Alexander II. im Kontext: Handlungsmuster soldatisch-aristokratischer Tugendhaftigkeit im europäischen Vergleich}

Der Vergleich mit anderen Staatsoberhäuptern in Europa zeigt, dass das Verhalten Alexanders II. nicht so ungewöhnlich war, wie es dem Publizisten Stanislas M. Propper seinerzeit erschien. Eine soldatisch-aristokratische Haltung bewies etwa auch Otto von Bismarck, als Ferdinand Cohen-Blind am 7. Mai 1866 versuchte, ihn zu erschießen. Den Zeitungsberichten in der Neuen Preußischen Zeitung zufolge ging Bismarck am Nachmittag vom Palais nach Hause, als hinter ihm zwei Schüsse fielen. Er drehte sich um, bemerkte den Attentäter, ging „mit starken entschlossenen Schritten auf denselben“ los und ergriff ihn am rechten Arm und an der Brust. Cohen-Blind gelang es jedoch seinen sechsläufigen Taschenrevolver in die linke Hand zu nehmen. Er gab zwei weitere Schüsse auf Bismarck ab, wobei er ihm die Pistole direkt auf die Brust setzte. Die Schüsse streiften jedoch ab. Der Bericht eines unbekannten Augenzeugen in der Neuen

13 Zur Bedeutung der Begegnung des Zaren mit seinem Volk für die Inszenierung des Herrschaftsideals von Alexander II. vgl. Richard Wortman, Scenarios of Power. Myth and Ceremony in Russian Monarchy. From Peter the Great to the Abdication of Nicholas II., Princeton 2006, S. 191.

14 Vgl. Michail Frolenko, Načalo narodovol'čestva, in: Katorga i ssylka 24. 1926, S. 17 -26, hier S. 21 und dazu Ulam, In the Name of the People, S. 345.

15 Stanislas M. Propper, Was nicht in die Zeitung kam. Erinnerungen des Chefredakteurs der „Birschewyja Wedomosti“, Frankfurt 1929, S. 100. 
Preußischen Zeitung fährt fort: „Graf Bismarck bewahrte eine bewundernswürdige Ruhe. Er richtete an den Mörder die Frage: ,Wer sind Sie und wo sind Sie her?" - welche Frage aber nicht beantwortet wurde." Zwei Polizisten nahmen den Attentäter in Gewahrsam, während Bismarck weiter nach Hause ging. ${ }^{16}$ Den Erinnerungen des Hauslehrers zufolge wartete seine Familie dort mit dem Essen. Der Ministerpräsident erfüllte jedoch erst seine Pflicht: Er schrieb in seinem Kabinett eine Notiz an den König und führte der Etikette gemäß die Gräfin von Stolberg zu Tisch. „Dann erst wandte er sich zu seiner Frau, küsste sie auf die Stirn und sagte: , Mein Kind, heute haben sie auch auf mich geschossen, aber es ist nichts. ${ }^{\text {}} 17$ Ob diese Worte nun so gesprochen oder im Nachhinein erfunden wurden, weil sie idealerweise so hätten gesprochen werden müssen, erscheint nicht von zentraler Bedeutung. Sie bringen das Muster des soldatisch-aristokratischen Umgangs mit Attentaten auf den Punkt.

Ein solch stoisches Verhalten im Umgang mit der Gefahr lud zu Wiederholungstaten ein. Der deutsche Kaiser Wilhelm I. fuhr für gewöhnlich jeden Nachmittag eine Runde im Tiergarten spazieren. Am 11. März 1878 saß er mit seiner Tochter ins Gespräch vertieft im Fond einer offenen Kalesche als Unter den Linden der Klempnergeselle Max Hödel hinter einem Fuhrwerk hervortrat, das am Rande der Straße stand, und mit einem Revolver zwei Schüsse auf den Kaiser abgab. Wilhelm gab einem Offizier Anweisung, ihm Nachricht zu bringen, sobald der Täter gefasst sei, und befahl im Übrigen, nach Hause ins Palais zu fahren. Dass auf ihn soeben ein Attentat verübt worden war, hatte der Achtzigjährige in diesem Moment noch gar nicht verstanden. In den eigenhändigen Aufzeichnungen des Kaisers, die sich in seinem Nachlass im Brandenburgisch-Preußischen Hausarchiv finden, heißt es:

Ich war, trotzdem dass meine Tochter sehr bewegt war, doch nicht überzeugt, dass von einem Attentat die Rede sei, da die Groß Herzogin mir erst viel später erzählen konnte, wie sie den ganzen Vorgang gesehen habe, so dass mir nun erst klar wurde, dass von einem Verbrechen die Rede sein müsse! ${ }^{18}$

16 Neue Preußische Zeitung, 10.5.1866, zit. nach Julius Hans Schoeps, Bismarck und sein Attentäter. Der Revolveranschlag Unter den Linden am 7. Mai 1866, Frankfurt 1984, S. 17. Vgl. zum Verlauf dieses Attentats auf Bismarck ebendort auch die Beschreibungen anderer Augenzeugen, die die Version der Neuen Preußischen Zeitung in den wesentlichen Punkten bestätigen, sowie Lothar Gall, Bismarck. Der weiße Revolutionär, Frankfurt 1980, S. 357 f.

17 Zit. nach Schoeps, Bismarck und sein Attentäter, S. 22. Vgl. auch die Darstellung des Diplomaten Robert von Keudell: „Ehe man sich zu Tische setzte, küßte er seine Gemahlin auf die Stirn und sagte: ,Erschrick nicht, mein Herz, es hat jemand auf mich geschossen, ich bin aber durch Gottes Gnade unverletzt geblieben. “" Robert von Keudell, Fürst und Fürstin Bismarck. Erinnerungen aus den Jahren 1846 bis 1872, Berlin 1901, S. 261.

18 Geheimes Staatsarchiv Preußischer Kulturbesitz, Brandenburgisch-Preußisches Hausarchiv, Rep. 51, Nr. 851, Aufzeichnung Kaiser Wilhelms über das Hödel'sche Attentat, Berlin 11.5.1878. 
Doch auch die Erkenntnis, dass hier jemand nach seinem Leben getrachtet hatte, hielt ihn nicht davon ab, weiterhin täglich zur gleichen Zeit in offener Kalesche im Tiergarten spazieren zu fahren. Und so ergab sich für Dr. Carl Nobiling die Gelegenheit, am 2. Juni 1878 seinerseits einen Anschlag auf das Leben Wilhelms I. zu versuchen. Zu diesem Zwecke hatte er ein Zimmer Unter den Linden angemietet, an dem der Kaiser vorbeifahren würde. Um leichter zu treffen, benutzte er eine Schrotflinte. Mit zwei Ladungen traf er Wilhelm I. an Gesicht, Arm, Rücken und Schulter. Da Nobiling „nur“ Hasenschrot und Rehposten geladen hatte, überlebte Wilhelm I. jedoch und konnte nach längerer Kur sogar noch einmal die Regierungsgeschäfte übernehmen. Seine Spazierfahrten im offenen Wagen setzte er nach seiner Rückkehr fort. ${ }^{19}$

Die symbolische Bedeutung der Aufrechterhaltung solcher Gewohnheiten wird etwa am Beispiel Queen Victorias deutlich. Die Queen of the United Kingdom of Great Britain and Ireland und First Empress of India hatte in ihrem Leben mindestens sieben Anschläge auf ihre Person zu überstehen. Doch nicht allein hinsichtlich der Anzahl der Attentate stand Queen Victoria dem russischen Zaren kaum nach. Auch in der Hartnäckigkeit, mit der sie Sicherheitsmaßnahmen ablehnte und an ihren täglichen Routinen festhielt, glich sie dem russischen und dem preußischen Monarchen. So lehnte sie die Verwendung eines mit Kettenhemd gepanzerten Schirms, den man nach dem ersten Attentat extra für sie hatte anfertigen lassen, rundweg ab und ließ sich nicht von ihren Spazierfahrten oder der Verwendung offener Kutschen abbringen. ${ }^{20}$

Eine Analyse der Presseberichterstattung verdeutlicht, warum Victoria in dieser Weise handelte. So heißt es in der Londoner Times über den 31. Mai 1842 - den zweiten Tag nach dem zweiten Anschlag von John Francis auf die

19 Zu den Attentaten Max Hödels und Dr. Carl Eduard Nobilings auf Kaiser Wilhelm I. vgl. nach wie vor Andrew R. Carlson, Anarchismus und individueller Terror im Deutschen Kaiserreich, 1870-1890, in: Wolfgang J. Mommsen u. Gerhard Hirschfeld (Hg.), Sozialprotest, Gewalt, Terror. Gewaltanwendung durch politische und gesellschaftliche Randgruppen im 19. und 20. Jahrhundert (= Veröffentlichungen des Deutschen Historischen Instituts London, Bd. 10), Stuttgart 1982, S. 207 -236. Vgl. auch die Darstellungen in der biographischen Literatur zu Wilhelm I., etwa Franz Herre, Wilhelm I. Der letzte Preuße, Köln 1980, S. 470 f. sowie aus sozialdemokratischer Sicht Franz Mehring, Geschichte der deutschen Sozialdemokratie, Teil 2: Von Lassalles „Offenem Antwortschreiben“ bis zum Erfurter Programm 1863 - 1891 [1898], Berlin 1980, S. 492 - 500. Für eine medienhistorische Interpretation siehe Carola Dietze, Von Kornblumen, Heringen und Drohbriefen. Ereignis und Medienereignis am Beispiel der Attentate auf Wilhelm I., in: Friedrich Lenger u. Ansgar Nünning (Hg.), Medienereignisse der Moderne, Darmstadt 2008, S. 40 - 60.

$20 \mathrm{Zu}$ den Attentaten auf Königin Victoria vgl. Giles Saint Aubyn, Queen Victoria. A Portrait, New York 1991, S. 160 - 164, S. 388 f. u. S. 421 sowie Christopher Hibbert, Queen Victoria. A Personal History, New York 2000. 
Queen, dass sich schon um acht Uhr morgens viele respektable Personen vor dem Buckingham Palace versammelt hätten. Ab zehn Uhr seien zudem die Kutschen der nobility und der gentry eingetroffen.

Towards 4 o'clock in the afternoon the crowd collected in front of the Palace continued rapidly to increase, in the anticipation that Her Majesty would, notwithstanding the fearful attempt at her destruction, still continue to take her accustomed airing, and we are happy to say the public anticipation was not disappointed. Precisely at 25 minutes to 5 o'clock the side gates at the north-eastern wing of the Palace were suddenly thrown open, and the Royal outriders appeared [...] preceding the Royal carriage (an open barouche) [...] which contained Her Majesty, the Prince Albert, and the Duke of Saxe Meiningen [...] The appearance of the Royal carriage emerging from the gateway was the signal for one long, loud and continued shout of hurrahs, accompanied by waving of handkerchiefs and of hats, and many were the loyal aspirations heard from the crowd of,God preserve the Queen'. Her Majesty, we rejoice to assure our readers, appeared perfectly calm and collected, although somewhat flushed. ${ }^{21}$

Die Botschaft von beiden Seiten ist deutlich: Während jede einzelne Person mit ihrer Präsenz und ihrem Jubel der Königin ihre Solidarität aussprach, und die Bevölkerung schon durch die schiere Masse der Erschienenen den Attentäter zu marginalisieren suchte, signalisierte Viktoria mit dem Festhalten an der nachmittäglichen Spazierfahrt im offenen Wagen, dass sie sich von den Attentaten nicht im mindesten einschüchtern ließ, und zeigte, dass solche Anschläge auf ihr Leben ihr nichts anhaben konnten. Explizit wird dies in einem Wortwechsel, der aus dem Jahre 1850 überliefert ist, nachdem Robert Pate ihr mit einem Stock ins Gesicht geschlagen hatte. Für den Abend dieses Tages war ein Opernbesuch vorgesehen, doch legte man ihr angesichts der Verletzungen nahe, lieber zu Hause zu bleiben. Dagegen soll die Queen eingewandt haben: „If I do not go, it will be thought I am seriously hurt.“ „But you are hurt Ma'am.“ „Very well, then everybody shall see how little I mind it. “22 In beiden Fällen wusste die Queen um die öffentlichen Erwartungen an ihr Verhalten und verhielt sich dazu entsprechend.

Das Ausmaß und die Bedeutung der öffentlichen Aufmerksamkeit und Kommentierung, lässt sich exemplarisch an der medialen Berichterstattung über das Attentat des Leipziger Studenten Oskar Wilhelm Becker auf Wilhelm I. zeigen. Becker gab mit einem Doppelterzerol zwei Schüsse auf den preußischen Monarchen ab, als dieser am Morgen des 14. Juli 1861 in der Lichtenthaler Allee bei Baden-Baden seiner Frau bei ihrem morgendlichen Spaziergang entgegenging. Bei der Festnahme des Attentäters mahnte Wilhelm I. wiederholt, dem jungen Mann nichts zuleide zu tun. Dann setzte er seinen Gang allein fort, traf seine Gemahlin, erwähnte ihr gegenüber den Vorfall aber gar nicht. Erst als ihm bei der Rückkehr nach Baden-Baden verschiedene

21 Attempt On The Life Of Her Majesty, in: The Times, 1.6.1842, S. 6.

22 Zit. nach Saint Aubyn, Queen Victoria, S. 164. 
Personen ihre Glückwünsche zur Errettung aus der Gefahr aussprachen und so eine Erklärung unausweichlich machten, soll er seiner Frau in scherzendem Ton gesagt haben: „Man hat etwas auf mich geschossen“. ${ }^{23}$ In den folgenden Tagen wurde dieses Verhalten des Königs in der deutschen Presse wie auch in Zeitungen des europäischen Auslands ausführlich beschrieben und kommentiert: man betonte die „wahrhaft Königliche Haltung Sr. Majestät nach dem Attentat“, die „Kaltblütigkeit“, „Ruhe und Milde“ mit der er den Attentäter behandelte und gab der Bewunderung Ausdruck über die „vollkommene Fassung und gleichmüthig heitere Haltung, mit welcher er die Damen und seine Umgebung zu beruhigen bemüht war. ${ }^{\text {¿24 }}$ Auch dass Wilhelm I. schon am gleichen Abend wieder mit seiner Tochter spazieren fuhr und die Königin von Preußen am nächsten Morgen wieder ihren gewohnten Spaziergang in der Lichtenthaler Allee unternahm und dabei demonstrativ auch die Stelle passierte, an der das Attentat geschehen war, wurde eigens gemeldet. ${ }^{25}$

Die Demonstration von Mut, Entschlossenheit und Milde sowie die demonstrative Missachtung von Gefahren war folglich nicht nur eine persönliche Angelegenheit, sondern von politischer Relevanz. Die Aufrechterhaltung der Ordnung, Rhythmen und Gewohnheiten am Hofe symbolisierte die Stabilität der politischen und gesellschaftlichen Ordnung. Wenn Attentäter und die von

23 Vgl. zum Hergang und zur Reaktion des Königs die verschiedenen Zeugenberichte im Generallandesarchiv Karlsruhe 250/8. Die Äußerung gegenüber Königin Augusta wird überliefert in Baden-Baden, 17. Juli, in: Kölnische Zeitung, 19.7.1861, S. 2; Das Attentat gegen Se. Majestät den König von Preußen, in: Wiener Zeitung, Abendblatt, 19.7.1861, S. 653; Au rédacteur, Bade, le 15 juillet 1861, in: Journal des Débats, 18.7.1861, S. 1 sowie Le Temps, 19.7.1861, S. 1.

24 Neue Preußische Zeitung, 17.7.1861, S. 1; Augsburger Allgemeine Zeitung, 16.7.1861, S. 3207; Das Attentat in Baden-Baden, in: Augsburger Allgemeine Zeitung, 17.7.1861, S. 3225 f., hier S. 3226 und wortgleich Das Attentat gegen Se. Majestät den König von Preußen, in: Wiener Zeitung, Abendblatt, 18.7.1861, S.649 f.; Vossische Zeitung, 17.7.1861, S. 3 und wortgleich Neue Preußische Zeitung, 17.7.1861, S. 1 sowie Kölnische Zeitung, 17.7.1861, S. 1. Vgl. darüber hinaus etwa die teilweise ausführliche Berichterstattung in: Extrablatt der Vossischen Zeitung, 15.7.1861; Vossische Zeitung, 16.7.1861, S. 2 und ebd., 18.7.1861, S. 3; Neue Preußische Zeitung, 16.7.1861, S. 1; Nationalzeitung, Morgenausgabe, 18.7.1861, S. 1 f.; Das Attentat auf Seine Majestät den König, in: Kölnische Zeitung, 17.7.1861, S. 2 sowie unter der Rubrik „Deutschland“ am 18. und 19.7.1861, S. 1; Das Attentat gegen Se. Majestät den König von Preußen, in: Wiener Zeitung, Abendblatt, 19.7.1861, S. 653 f.; Au rédacteur, Bade, le 15 juillet 1861, in: Journal des Débats, 18. 7.1861, S. 1; Le Temps, 19.7. 1861, S. 1; The Times, London, 19.7. 1861, S. 12; The Manchester Daily Examiner and Times, 19.7.1861, S. 4; Sanktpeterburgskija vedomosti, 8./20.7.1861, S. 1 und Severnaja pčela, 11./23.7.1861, S. 623.

25 Vgl. etwa Vossische Zeitung, 16.7.1861, S. 2; Neue Preußische Zeitung, 17.7.1861, S. 1; Nationalzeitung, Morgenausgabe, 18.7.1861, S. 2; Kölnische Zeitung, 17.7.1861, S. 3 und Au rédacteur, Bade, le 15 juillet 1861, in: Journal des Débats, 18.7.1861, S. 1. 
ihnen verübten Anschläge den Monarchen dazu zwingen konnten, den Tagesrhythmus umzustellen oder ihre Bewegungsfreiheit einzuschränken, dann war das dieser Logik zufolge ein Signal, dass die staatliche und gesellschaftliche Ordnung gefährdet war. Die Missachtung der von Attentätern ausgehenden Gefahren war deshalb ein staatspolitischer Akt, der von der Gesellschaft honoriert, ja geradezu eingefordert wurde. Der Artikel aus der Times sowie die Berichterstattung in der deutschen und internationalen Presse zeigen dies deutlich. Die Unfähigkeit oder der Unwillen, dieser Erwartung zu entsprechen, hätte - so darf man annehmen - dem Ansehen der Monarchen und damit auch dem Ansehen der jeweiligen Monarchie ernsthaft geschadet.

Bei dem Attentat Beckers auf Wilhelm I. war die Haltung des Monarchen über diese Symbolik hinaus jedoch in einem noch unmittelbareren Sinne von politischer Bedeutung. Dies wird an der politischen Deutung der Reaktion des Monarchen in der nationalen und internationalen Presse deutlich:

Der König hatte sich nach dem Attentat rasch gefasst und bewahrte eine ruhige Haltung. Nach der ersten Begrüßung sprach die Großfürstin Helene ein kluges und tapferes Wort; noch weinend sagte sie dem Könige: es werde nicht fehlen, dass man dieses Verbrechen auszubeuten suche, aber solche Bemühungen werde gewiß seine Entschlossenheit vereiteln. ${ }^{26}$

Während eine Reihe von liberalen Zeitungen in Deutschland auf diese Weise ihre Hoffnungen der Großfürstin Helene in den Mund legten, wurde man in Frankreich deutlicher. So schrieb Le Temps:

Le caractère ferme et honnête du roi, son vigoureux bon sens donnent le droit d'espérer que la cause libérale n'aura pas à pâtir de cet acte insensé et criminel; et que le coup de pistolet de l'allée de Lichtenthal [...] ne sera pas le signal d'une réaction en Allemagne. ${ }^{27}$

Die Zeitungskommentatoren sahen folglich einen engen Zusammenhang zwischen der unmittelbaren persönlichen Reaktion des Königs nach dem Attentat und der zu erwartenden politischen Reaktion der Regierung. „Muth und Gottvertrauen“ des „ritterlichen König“ boten - so hofften zumindest die Liberalen - die Gewähr dafür, dass „unsere Tage [...] die Unheilsthat nicht zur Quelle neuen Unheils machen" würden, ${ }^{28}$ und das bedeutete: dass sich Maßnahmen wie die Karlsbader Beschlüsse nicht wiederholen sollten und das Land von einer erneuten Demagogenverfolgung verschont bliebe.

Neben diesen allgemeinen Zügen des Umgangs mit Attentaten durch Staats-

26 Das Attentat auf Seine Majestät den König, in: Kölnische Zeitung, 17.7.1861, S. 2. Wiederabdruck in: Vossische Zeitung, 18.7.1861, S. 3 u. Nationalzeitung, Morgenausgabe, 18.7.1861, S. 2.

27 Attentat sur la personne du Roi de Prusse, in: Le Temps, 18.7.1861, S. 1.

28 Karlsruher Zeitung, 17.7.1861, zit. nach Kölnische Zeitung, 19.7.1861, S. 2, auszugsweise abgedruckt auch in Sanktpeterburgskija vedomosti, 12./24.7.1861, S. 878. Vgl. mit gleicher Logik, aber anderer politischer Ausrichtung auch Neue Preußische Zeitung, 17.7.1861, S. 1. 
und Regierungsoberhäupter im 19. Jahrhundert, gibt es jedoch auch signifikante Unterschiede zwischen der Reaktion Bismarcks, des jungen Wilhelm I. sowie des Zaren einerseits und dem Verhalten Queen Victorias sowie des alten Wilhelm I. andererseits: Während der junge Wilhelm I., Bismarck und Alexander II. ihre Attentäter mit ihrer - in der Tat jeweils sehr stattlichen und imposanten - Person direkt konfrontierten, verzichteten Victoria und der greise Wilhelm I. auf solche Begegnungen. Beim alten Kaiser liegen die Umstände dabei insofern etwas eigentümlich, als er die Schüsse Hödels zunächst nicht als Attentat interpretierte und Nobilings Schrotladungen ihn so sehr verletzten, dass er direkt zum Palais zurückgefahren werden musste. Zudem spielte hier sicher das Alter des Monarchen eine Rolle.

Aufschlussreich ist jedoch der Vergleich mit Victoria, denn er verweist auf die Bedeutung einer anderen Kategorie: der des Geschlechts. Der Londoner Times zufolge zog Prince Albert, der am Tag des ersten Anschlags auf die Queen im Juni 1840 neben Victoria in der Kutsche saß, die Königin, die gerade im dritten Monat schwanger war, zu sich, warf seine Arme schützend um sie und befahl dem Kutscher weiterzufahren, so dass auch der zweite Schuss fehlging. ${ }^{29}$ Was für Alexander II. undenkbar gewesen wäre oder wie im Falle Komissarovs als direkter Eingriff Gottes gedeutet werden musste, nämlich die Intervention von Personen zu seinem Schutz, war für Victoria möglich. Der Schutz einer Frau war eben selbstverständlich Männersache. Ja mehr noch: In ihrem Falle konnte Prince Albert kurzfristig das Kommando übernehmen, um sie aus der Gefahrenzone zu bringen, ohne dass damit für die Queen of the United Kingdom ein Ansehensverlust zu befürchten war; dagegen wäre eine Konfrontation mit dem Attentäter der schwangeren Königin vermutlich als in hohem Maße leichtsinnig und verantwortungslos vorgehalten worden. Demgemäß war ihre Empörung über die Attentate geschlechtsspezifisch aufgeladen. Nach dem vierten Anschlag 1850 schrieb sie in ihr Tagebuch:

Certainly it is very hard \& very horrid that I, a woman - a defenceless young woman \& surrounded by my children should be exposed to insults of this kind, and be unable to go out quietly for a drive. [...] For a man to strike any woman is most brutal. ${ }^{30}$

Waren die Anschläge für Victoria schon auf Grund ihres Geschlechts unziemliche Übergriffe, mussten sie für den jungen Wilhelm I., Bismarck und Zar Alexander II. Herausforderungen sein, denen man sich zu stellen hatte.

29 Atrocious Attempt to Assassinate the Queen and Prince Albert, in: The Times, 11.6.1840, S. 4.

30 Zit. nach Hibbert, Queen Victoria, S. 424. 


\section{Gleichzeitigkeit der Ungleichzeitigkeit: Traditionelle Herrscher in moderner Gefahr}

Dass sich das Festhalten an traditionellen Verhaltensmustern soldatisch-aristokratischer Tugendhaftigkeit gegen Ende des 19. Jahrhunderts zu einem für Leib und Leben europäischer Regenten gefährlichen Anachronismus entwickeln sollte, hatte vor allem mit neuen Formen persönlichen Risikos zu tun, denen sich die Staatsoberhäupter im Industriezeitalter zunehmend ausgesetzt sahen. Sicherheit von Herrschern war angesichts der Gefahr durch terroristische Anschläge immer weniger eine Frage männlicher Standhaftigkeit sondern der zunehmenden Technisierung und Entpersonalisierung von Schutzmaßnahmen. Dass dieser Anpassungsprozess von Sicherheitsbewusstsein an neue Formen der Gefahr nur schleppend verlief, lässt sich besonders gut am russischen Beispiel aufzeigen. Gerade hier schienen nicht nur die Gegner des autokratischen Regimes in hohem Maße aufgeschlossen gegenüber neuen Möglichkeiten des terroristischen Kampfes gewesen zu sein. Auch hielten die russischen Selbstherrscher länger als andere an überkommenen Verhaltensmustern und der Überzeugung vom göttlichen Schutz ihrer Dynastie fest.

\section{Challenge: Terroristen und der Fortschritt}

Die Geschichte der Herrscherattentate im Russischen Reich in der zweiten Hälfte des 19. Jahrhunderts - vom ersten Mordversuch durch Karakosov im Jahr 1866 bis zum „erfolgreichen“ Bombenanschlag der Gruppe Narodnaja volja am 1. März 1881 - lässt sich zum einen als eine Entwicklung von der Tat eines Einzeltäters zur geplanten Aktion einer terroristischen Gruppe beschreiben. Zum anderen fällt die zunehmende Technisierung politisch motivierter Gewalt in diesem Zeitraum ins Auge. Hatte Karakosov am Zaun des Sommergartens in St. Petersburg noch einen einfachen Revolver auf Alexander II. gerichtet, griffen die Aktivisten des „Volkswillens“ ab den 1870er Jahren verstärkt auf moderne Mittel des terroristischen Kampfes zurück und setzten zugleich auf die öffentliche Wirkung einer spektakulären Aktion im beginnenden Medienzeitalter. Insbesondere die Erfindung des Dynamits sollte die Geschichte des Terrorismus in Russland in der zweiten Hälfte des 19. Jahrhunderts maßgeblich prägen. Der 1866 von Alfred Nobel entwickelte Sprengstoff zog die Aktivisten der Organisation Narodnaja volja frühzeitig in den Bann. Sieben Anschlagsversuche unternahmen die Terroristen mit Dynamit, ehe im achten Anlauf am 1. März 1881 Kaiser Alexander II. getötet wurde. Während die loyalen Diener des Zarenregimes von den „so genannten,wissenschaftlich“ ausgeführten Mordversuchen [...] mit Hilfe der furchtbarsten Sprengstoffe in künstlichen Bomben und Minen, die durch die Chemiker der Partei verfertigt wurden “31, schockiert

31 Richard von Pfeil und Klein-Ellguth, Das Ende Kaisers Alexander II. Meine Erlebnisse in russischen Diensten 1878 -1881, Berlin 1901, S. 53. 
waren, berichtete Vera Figner, ein Mitglied des Exekutivkomitees von Narodnaja volja, begeistert in ihren Memoiren:

Es war ein langer Weg der Perfektionierung der Vorrichtungen und der Sammlung von Erfahrung von der ersten Idee [Dynamit einzusetzen] und dem Bau der ersten primitiven Apparate bis [zum Einsatz] dieser eleganten und handlichen Sprengkörper, die von den Aktivisten der Narodnaja volja Isaev und Kibal'čič für den [Anschlag vom] 1. März 1881 entwickelt worden waren. ${ }^{32}$

Eine weitere Errungenschaft des modernen Zeitalters, deren Nützlichkeit relativ früh von den oppositionellen Kräften entdeckt wurde, war die Eisenbahn, deren Bau im Zarenreich seit den 1850er Jahren von staatlicher Seite vorangetrieben wurde. Bereits 1865 deckte die III. Abteilung der Kanzlei seiner Kaiserlichen Majestät, d.h. die Geheimpolizei des Zarenregimes, den Plan einer Gruppe polnischer Aktivisten auf, die einen Anschlag auf die Zugstrecke von Warschau nach Moskau vor der Reise Alexanders II. mit seinem Hofzug planten. ${ }^{33}$ Im Oktober 1869 erreichte die Polizei von Elizavetgrad ein anonymer Brief, der von den Plänen zweier junger Personen berichtete, einen Bombenanschlag auf den Hofzug Alexanders II. zu verüben. ${ }^{34}$ Den Behörden gelang es zwar, diese Anschläge zu vereiteln, aber es schien nur eine Frage der Zeit zu sein, ehe eine andere Gruppe versuchte, den Zaren während einer Eisenbahnreise zu ermorden. Die Eisenbahn stellte aus zwei Gründen ein ideales Ziel politischer Attentate dar. Zum einen war es den staatlichen Sicherheitsorganen nahezu unmöglich, das wachsende Schienennetz Tag und Nacht gänzlich zu überwachen. Aktivisten eröffnete sich dadurch die Möglichkeit, Strecken, die der Hofzug des Kaisers befahren würde, im Vorfeld zu beschädigen oder mit einem Sprengsatz zu präparieren. Zum anderen konnten sich Terroristen jene physikalischen Kräfte zunutze machen, die sich entfalteten, wenn ein Zug in voller Fahrt entgleist oder abrupt zum Stillstand gebracht wird. Wenn es gelang, den Gleiskörper vor der Durchfahrt des Hofzuges

32 Vera Figner, Polnoe sobranie sočinenij v 6 tomach, Bd. 1, Moskau 1928, S. 122. Zu den ersten Experimenten der Gruppe Narodnaja volja mit Dynamit vgl. Wera Figner, Nacht über Russland, Berlin 1928, S. 101. Von den technischen Fähigkeiten der Terroristen waren auch die Repräsentanten der Autokratie beeindruckt. Die Hinrichtung des Technikers Kibal'čič soll der Generalgouverneur von Odessa, General Eduard Totleben, mit der Bemerkung kommentiert haben, einen so begabten Mann solle man nicht töten, sondern in ein Labor sperren und sein Talent in den Dienst der Regierung stellen. Vgl. Jörg von Uthmann, Attentat. Mord mit gutem Gewissen, Berlin 1996, S. 103.

33 Gosudarstvennyj Archiv Rossijskoj Federacii (im folgenden GARF), f. 109, op. 2a, ed. chr. 788, 1. 1-3ob.

34 Philipp Pomper, Sergei Nechaev, New Brunswick 1979, S. 97, ders., Nechaev and Tsaricide. The Conspiracy within the Conspiracy, in: Russian Review 33. 1974, S. $123-138$, hier S. 125. 
zu manipulieren, konnte nicht nur damit gerechnet werden, dass der Zug aus den Schienen sprang, sondern zudem damit, dass die Waggons dem Aufprall nicht standhielten und deren Insassen in den Trümmern ernsthaft verletzt oder gar getötet werden würden.

Den ersten Anschlag auf Alexander II., bei dem nach der Verhängung des "Todesurteils“ über den Reformzaren im Sommer 1879 sowohl die Sprengwirkung von Dynamit als auch die physikalischen Kräfte eines Eisenbahnunfalls ausgenutzt werden sollten, planten die Aktivisten von Narodnaja volja im Winter 1879. An drei Punkten, die der Hofzug Alexanders II. am 18. bzw. 19. November auf seiner Rückfahrt von der Krim nach Moskau passieren sollte, versuchten die Aktivisten der Organisation im Vorfeld Bomben zu installieren, die sie kurz vor der Durchfahrt des Zarenzuges detonieren lassen wollten. ${ }^{35}$ Die Vorbereitungen am ersten Schauplatz in der Nähe der Stadt Odessa wurden relativ bald aufgrund einer veränderten Reiseroute des Zaren wieder aufgegeben. Am zweiten Punkt auf der Lovozo-Sevastopol'-Bahn scheiterte der geplante Anschlag, weil einer der Aktivisten die Elektroden der Bombe nicht sachgemäß verbunden und dadurch die Zündung des Sprengsatzes verhindert hatte. Auch am dritten Ort, an der Bahn von Kursk nach Moskau, entkam Alexander II. der Detonation. Stepan Šriaev, der ein kleines Haus in der Nähe eines Bahnhofs bei Moskau bezogen hatte, zündete die Bombe erst, nachdem der erste der beiden Hofzüge den entsprechenden Abschnitt bereits passiert hatte. Im zweiten Zug, der nach der Detonation entgleiste, reiste das kaiserliche Gefolge. Trotz der Explosion und der Entgleisung des Zuges wurde niemand bei diesem Anschlag getötet. Ungeachtet des „Fehlschlags“ war die Gruppe stolz auf Planung, Größe und Aufwand dieses Attentats. Das Hausjournal der Gruppe vom 1. Januar 1880 verkündete selbstbewusst, frühere Versuche, den Zaren zu ermorden, seien vergleichsweise primitiv gewesen: „Immer stellte sich ein Mann mit einer Pistole dem Herrscher über Millionen von Angesicht zu Angesicht gegenüber. [Der Attentäter] hatte keine Chance zu entkommen und [mit seiner Tat] nur geringe Aussichten auf Erfolg." Wie sehr unterschied sich davon der Anschlag vom 19. November! „Das Attentat, war gründlich geplant und vorbereitet - eine

35 Zum Anschlag vom 19.11.1879 vgl. u.a. Ulam, In the Name of the People, S. 336 ff.; Stepan Stepanovič Volk, Narodnaja volja. 1879-1882, Moskau 1966, S. 100 - 102; Figner, Nacht über Russland, S. 121 - 125; dies., Polnoe sobranie sočinenij v šesti tomach, Bd. 1, Moskau 1928, S. 183-189; Ernst R. Rutkowski, Die revolutionäre Bewegung und die inneren Verhältnisse des Zarenreiches von 1877 bis 1884 im Urteil österreichisch-ungarischer Diplomaten, in: Mitteilungen des Österreichischen Staatsarchivs 9. 1956, S. 348 - 483, insbes. S. $394-397$; Nabroskij vospominanij knjazja D[mitrija] D. Obolenskogo, in: Russkij Archiv, 1894, Nr. 10, S. 251 - 286, insbes. S. 270-279; Političeskaja policija i političeskij terrorizm v Rossii. Vtoraja polovina XIX - načalo XX vv., Sbornik dokumentov, hg.v. Gennadij A. Bordjugov, Moskau 2001, S. 48-53 u. S. 84-93. 
beträchtliche Geldsumme, Arbeitskraft und fundierter technischer Sachverstand kamen zum Einsatz. ${ }^{\text {“36 }}$ Selbstsicher prahlte die Organisation:

Die zunehmende Perfektionierung der Kampfmethoden erfüllt uns mit überaus großer Befriedigung. [...] Die ,Autodidakten' [nedouckki] haben sich die besten Waffensysteme angeeignet und bringen die letzten und neuesten wissenschaftlichen Errungenschaften auf dem Gebiet der Technik zum Einsatz. In allen Phasen des Kampfes erinnern die Revolutionäre unwillkürlich an die höchste kulturelle Rasse [vysšaja kul'turnaja rasa], die ihre Kräfte mit den zahlreichen, aber wilden Horden der Regierung misst. Das Wissen und die Erfindungsgabe sind zweifelsohne auf Seiten der ,Halbgebildeten' ${ }^{37}$

„Mit Chemie und Elektrizität als Gehilfen hatte der Revolutionär den Zarenzug gesprengt“, berauschte sich auch Vera Figner später an den neuen Kampfmethoden. Zwar sei der Anschlag von 1879 ein „Misserfolg“ gewesen. Er habe jedoch einen „ungeheuren Eindruck in Russland hervor [gerufen] und Widerhall in ganz Europa [geweckt]. ${ }^{\text {38 }}$ Der Anschlag an sich - als organisatorische Großleistung und Medienereignis - hatte das Attentat als Mittel zum Erreichen etwaiger politischer Ziele (wie illusionär diese auch immer sein mochten) inzwischen weit in den Schatten gestellt. Gegen solch eine Begeisterung für die Gewalt der Technik und das Spektakel der Destruktion war mit zwischenmenschlicher Konfrontation nach dem Ehrenkodex soldatisch-aristokratischer Tugendhaftigkeit wenig auszurichten.

Die russische Öffentlichkeit war von den neuen Kampfmethoden des unsichtbaren Feindes hingegen zutiefst schockiert. Zwei Tage nach dem Anschlag kommentierte die Zeitung Russkie vedomosti:

Die angewandten Kampfmethoden zeigen, dass [die subversiven Elemente] einen wagemutigen und schrecklichen Schritt nach vorne getan haben. Die Waffen, die sie in der Vergangenheit benutzten, scheinen ihnen nicht mehr auszureichen. Dolche und Pistolen, die für einen Augenblick einer fanatischen Hand bedürfen, wurden weggeworfen, weil sie nicht zu dem erhofften Ergebnis geführt haben. Sie wurden durch langsame Aktionen ersetzt, die sowohl eine dauerhafte Anspannung als auch bösartige Handlungen erfordern. [Die subversiven Elemente] sind von der Erdoberfläche in den Untergrund verschwunden, von wo sie ihre unheilbringenden Aktivitäten ausführen. ${ }^{39}$

\section{Einen Tag später ergänzte eine Petersburger Zeitung:}

Kriminelle Handlungen dieser Art übersteigen die Vorstellungsmöglichkeiten der unverdorbenen und jungfräulichen Phantasien des einfachen Volkes. [Ein Vorfall dieser Art] wäre nie passiert, wenn die Bevölkerung eine solche Tat für möglich gehalten hätte. Wenn man den Bewohnern Moskaus gesagt hätte, dass jemand möglicherweise Eisenbahngleise untertun-

36 Narodnaja volja. Social'no-revoljucionnoe obozrenie, 1.1.1880, S. 4, zit. nach dem Quellenband Literatura partii „Narodnaja volja“, Moskau 1930, S. 48.

37 Ebd., S. 49.

38 Figner, Nacht über Russland, S. 127 u. S. 124.

39 Russkie vedomosti, Nr. 294, 20.11.1879, S. 1. 
nelt habe, um dort eine Bombe zu zünden, wenn der Hofzug des Kaisers vorbeifährt, wäre jeder Russe losgezogen, um alle Häuser und Hütten in der Nähe von Eisenbahnanlagen zu untersuchen und um sich persönlich zu vergewissern, dass nichts [und niemand] die Ruhe des Monarchen bedroht. ${ }^{40}$

Damit suggerierte die Zeitung, dass die Einheit von Zar und Volk auch in der Lage sei, den Gefahren des modernen Terrorismus zu trotzen.

Die glückliche Rettung des Zaren wurde von der Öffentlichkeit schließlich als ein Zeichen der Gnade Gottes interpretiert, der „erneut das teure Leben des Monarchen gerettet hatte “. ${ }^{41}$ Bald verbreitete sich das Gerücht, dass der Zar ursprünglich geplant hatte, im zweiten Zug zu reisen, der dem Bombenanschlag der Terroristen zum Opfer gefallen war. Der Zar sei jedoch, so diese Version der Geschichte, früher als vorgesehen am Ort der Abfahrt in Simferopol eingetroffen, woraufhin die Fahrpläne der Züge des Kaisers und des Gefolges ausgetauscht worden seien. ${ }^{42}$ Diese Episode konnte auch als eine Form göttlicher Intervention in die von Menschen erdachten Rhythmen des modernen Lebens interpretiert werden, die von Fahrplänen und technischen Betriebsabläufen bestimmt wurden: Durch seinen Stellvertreter auf Erden griff Gott in die arrangierte Ordnung ein und durchkreuzte so die heimtückischen Pläne der Terroristen. Diese Version der Geschichte wurde durch die Pointe abgerundet, dass Zar Alexander II., der im ersten Zug gereist war, im Moment der Detonation in Moskau vor einer wundertätigen Ikone der Mutter Gottes gebetet haben soll. ${ }^{43}$ Dieser Lesart der Ereignisse folgend stellte eine Agenturmeldung vom 20. November 1879 die Rettung des Zaren als (göttliches) "Wunder" (čudo) dar. ${ }^{44}$ Am selben Tag hatte auch Alexander II. in seiner

40 Sankt Peterburgskie vedomosti, 22.11.1879, S. 1.

41 Rasskaz očevidca o vzryve na Moskovsko-Kurskoj železnoj doroge, in: Saratovskie eparchal'nye vedomosti, 1879, Nr. 44, S. 376-378, hier S. 377, Wiederabdruck aus den Moskovskie vedomosti; Vladimir Petrovič Meščerskij, Moi vospominanija. Čast vtoraja, 1865 - 1881, St. Peterburg 1898, S. 438. Vgl. auch: Denkwürdigkeiten des Botschafters Generals von Schweinitz, Bd. 2, Berlin 1927, S. 83. Schon die Rettung Alexanders II. vor den Schüssen Karakosovs am 4.4.1866 am Petersburger Sommergarten interpretierten die Zeitgenossen als ein Zeichen göttlicher Intervention. Bei einem Empfang der Großfürsten und anderer Mitglieder des Hofes am Mittag des gleichen Tages hob Alexander in seiner kurzen Ansprache hervor, dass ihn am Vormittag „Gottes Gnade errettet" habe. Vgl. von Pfeil und Klein-Ellguth, Das Ende Kaisers Alexander II., S. 35. Aus Dankbarkeit wurde an der Stelle der Rettung des Zaren am Zaun des Sommergartens eine Kapelle zu Ehren seines Schutzheiligen Alexander Nevskij errichtet.

42 Berichte eines Mitarbeiters der militärischen Postkanzlei (voenno-počtovaja kanceljarija) und des Fürsten Dmitrij D. Obolenskij, die beide im zweiten Zug reisten: Rasskaz očevidca o vzryve, S. 377; Nabroskij vospominanij knjazja D[mitrija] D. Obolenskogo, S. 270. Vgl. auch Dnevnik D[mitrija] A. Miljutina. 1878-1880, Bd. 3, Moskau 1950, S. 179.

43 Von Pfeil und Klein-Ellguth, Das Ende Kaisers Alexander II., S. 54.

44 Vestnik Evropy 14. 1879, Bd. 12, S. 857. 
Ansprache vor den Würdenträgern Moskaus im Georg-Saal des Kremls betont, dass „Gott Mich und alle, die mit Mir nach Moskau fuhren, [erneut] beschützt hat. “" ${ }^{\text {“5 }}$ Nach der Rettung des Kaisers war in St. Petersburg die Reproduktion eines Gemäldes „massenhaft“ im Umlauf, das die „göttliche Beschirmung des Zaren bei diesem Mordversuch“ zeigte:

Man sieht den Verbrecher hinter der Wand eines einsamen Hauses stehend, den herannahenden ersten Zug aufmerksam beobachten. Über dem mittelsten Wagen dieses Zuges schwebt ein Engel, welcher seine Hände wie beschützend über demselben ausbreitet. Der Vorgang ist bei Abendlicht dargestellt und der helle Schein der Lokomotivlaternen beleuchtet den Engel mit glänzenden Strahlen. ${ }^{46}$

Aus den Memoiren Vera Figners und denen anderer Zeitgenossen wissen wir jedoch, dass die wundersame Rettung des Zaren am 19. November 1879 vermutlich profanere Gründe hatte. Tatsächlich reiste der Zar in Simferopol fahrplanmäßig mit dem zweiten Zug ab, was die lokalen Verbindungsleute der Organisation Narodnaja volja mit folgender verschlüsselter Botschaft nach Moskau telegrafierten: „Wir haben Großmutter in der Früh zum Zug gebracht. Holt sie ab. Der Weizen kostet zwei Rubel, unser Preis: 4 Rubel“, sprich: der Zar reist im vierten Waggon des zweiten Zuges. ${ }^{47}$ Während der Fahrt tauschten die beiden Züge jedoch die Positionen, was bei Reisen dieser Art nichts Außergewöhnliches war. $^{48}$ Wenn Stepan Širiaev den Zünder der Bombe eine Stunde früher aktiviert hätte, wäre die Bombe bereits unter dem Zug des Zaren detoniert mit all den unvorhersehbaren Folgen. ${ }^{49}$

Unmittelbar nach dem Anschlag berichtete die russische Presse detailliert über die Hintergründe der Tat. Am 27. November 1879 informierte die konservative Zeitung Novoe vremja ihre Leser über die Sicherheitsvorkehrungen, die man von staatlicher Seite im Vorfeld dieser Reise zum Schutz des Kaisers getroffen hatte. An beiden Seiten der Gleise hatte man im Abstand von ca. 25 Schritt Streckenposten aufgestellt, die über die illuminierte Strecke zu wachen hatten. ${ }^{50}$ Erst nach der Detonation der Bombe sei das Haus an der Unglücksstelle, in dem sich Stepan Širiaev und Sofija Perovskaja eingemietet

45 Ebd., S. 858. Auch Kriegsminister Dmitrij Miljutin spricht in seinem Tagebuch von einer „wundersamen Rettung des Herrschers“ (čudesnoe spasenie gosudarja), vgl. Dnevnik D. A. Miljutina, Bd. 3, S. 180.

46 Von Pfeil und Klein-Ellguth, Das Ende Kaisers Alexander II., S. 54.

47 Nabroskij vospominanij knjazja D. D. Obolenskogo, S. 271.

48 Vgl. Sergej Michailovič Serpokryl, Podvig pered kazn’ju, Leningrad 1971, S. 101.

49 Figner zufolge gab die Terroristin Sofija L'vovna Perovskaja bereits beim Herannahen des ersten Zuges ein entsprechendes Zeichen, auf das Stepan Širjaev, der den Zündmechanismus aktivieren sollte - aus ungenannten Gründen - jedoch nicht reagierte. Vgl. Figner, Polnoe sobranie sočinenij, Bd. 1, S. 188.

50 Podrobnosti pokušeniij 19-go nojabrja, in: Novoe vremja, 27.11.1879, Nr. 1347, (= GA RF f. 109, op. 1a, ed. chr. 984, 1.1-2); Rasskaz očevidca o vzryve, S. 377. 
und die Bombe gezündet hatten, von den Sicherheitskräften entdeckt worden. ${ }^{51}$ In einem anderen Artikel mutmaßt ein Augenzeuge, dass die Polizei im Haus auch dann nichts Verdächtiges gefunden hätte, wenn es im Vorfeld einer Untersuchung unterzogen worden wäre. ${ }^{52}$ Jedes Teil der unheilvollen Apparatur sei sorgfältig getarnt und versteckt gewesen. Die Presse war entsetzt über die Kaltblütigkeit, mit der die Terroristen einen 40 Meter langen Tunnel von ihrem Versteck bis zum Bahndamm gegraben und in diesem ein Zündkabel bis zur Bombe unter den Gleisen gelegt hatten. Voller Abscheu wurde in der Novoe vremja darüber berichtet, dass Širiaev und Perovskaja ihre nächtlichen Aktivitäten durch einen besonders religiösen Lebenswandel am Tage getarnt hätten. ${ }^{53}$ Nach dem Abschluss der polizeilichen Untersuchungen an der Unglücksstelle wurde das Haus, in dem sich die beiden Terroristen versteckt hatten, am 21. November 1879 von einem aufgebrachten Mob von ca. 4.000 Menschen gestürmt und völlig zerstört. ${ }^{54}$

\section{Response: Reaktion der Staatsoberhäupter und ihrer Sicherheitsorgane}

Der Anschlag auf den Zarenzug vom November 1879 führte den russischen Sicherheitsbehörden sowohl den technischen Entwicklungsstand des terroristischen Untergrunds als auch das sich daraus ableitende neue Gefahrenpotential für das Leben des Zaren deutlich vor Augen. Bereits nach dem Attentatsversuch Aleksandr Solov'evs auf den Zaren am 2. April 1879 und insbesondere nach dem Bombenanschlag auf den Hofzug am 19. November lässt sich eine deutliche Verschärfung der Sicherheitsbestimmungen für den Herrscher beobachten. So wurde z. B. die Mobilität des Regenten dadurch eingeschränkt, dass er und die Mitglieder der kaiserlichen Familie von nun an nur noch mit einer Eskorte durch St. Petersburg fahren durften. ${ }^{55}$ Auch

51 Offensichtlich gab es unter den Journalisten Verwirrung, welches Pseudonym der Käufer, vermutlich Lew N. Gartman (Hartmann), benutzt hatte, der das entsprechende Haus an der Strecke kaufte. Die Zeitung Novoe vremja berichtet, der Mann habe Suchorukov geheißen, die Moskovskie vedomosti hingegen berichteten hingegen von einem gewissen Kleinbürger Samarskij. Podrobnosti pokušeniij 19-go nojabrja, 1. 2; Rasskaz očevidca o vzryve, S. 378.

52 Rasskaz očevidca o vzryve, S. 378.

53 Podrobnosti pokušeniij 19-go nojabrja, 1. 2;

54 Ebd. Die Terrororganisation Narodnaja volja behauptete demgegenüber, dass die Bevölkerung Moskaus den missglückten Anschlag auf den Zaren mit großer Gleichgültigkeit aufgenommen habe. Vgl. Narodnaja volja. Social'no-revoljucionnoe obozrenie, 1.1.1880, in: Literatura partii „Narodnaja volja“, S. 58.

55 Propper: Was nicht in die Zeitung kam, S. 69. Auch am 1.3.1881 wurde die gepanzerte Kutsche des Kaisers auf der Fahrt entlang des Petersburger Katharinenkanals von bewaffneten Kosaken eskortiert. Bei dem Attentat auf Alexander II. wurde einer der Kosaken getötet und ein anderer verwundet. Vgl. Dnevnik D. A. Miljutina. 1881-1882, Bd. 4, Moskau 1950, S. 26. Zum Attentatsversuch vom 2.4.1879 vgl. v. Borcke, Gewalt 
seinen Morgenspaziergang unternahm Alexander II. seit diesem Zeitpunkt nur noch innerhalb der Mauern des Winterpalastes. ${ }^{56}$ Die Mitglieder der Romanov-Dynastie waren von den Maßnahmen, die zum Schutze ihrer körperlichen Unversehrtheit getroffen wurden, alles andere als angetan. „Kaiser Alexander III. sollen anfänglich alle jene Sicherheitsmaßregeln geradezu widerlich gewesen sein“, bemerkte ein Zeitgenosse. ${ }^{57}$ Schon als Großfürst hatte er sich innerlich gegen die Einschränkung seiner Bewegungsfreiheit in der Hauptstadt gesträubt. Seinem Tagebuch vertraute er am 6. April 1879 an:

Heute musste ich zum ersten Mal in einer Kutsche mit Begleitschutz fahren. Ich kann gar nicht sagen, wie schmerzhaft und schmachvoll dies für mich war! Von Kosaken eskortiert durch unser allzeit friedliches und ruhiges St. Petersburg zu fahren als herrschte Krieg ist einfach schrecklich und daran lässt sich nichts ändern. ${ }^{58}$

Zeitgenossen fiel jedoch auf, dass sich Alexander III. schließlich mit der veränderten Realität und den nötigen Sicherheitsvorkehrungen abgefunden hatte. Man hatte „ihm vorgestellt, dass sie nicht allein zu seiner persönlichen Sicherheit, sondern zur Erhaltung des Staates in seiner jetzigen Form notwendig seien. ${ }^{\text {"59 }}$ Die letzte Formulierung deutet darauf hin, dass sich auch bei den russischen Monarchen gegen Ende des 19. Jahrhundert langsam die Einsicht durchsetzte, dass der Schutz ihrer Person nicht mehr in erster Linie eine Frage der persönlichen Ehre, sondern zunehmend eine der Staatsräson war.

Bereits die „Instruktionen für den Verkehr des Zarenzuges“, die im November 1879 in Kraft waren, schrieben vor, dass die vom Hofzug des Kaisers zu befahrende Strecke auf beiden Seiten von Streckenposten zu bewachen sei, die am Tage im Abstand von 100 sažen (ca. 200 Meter), in der Nacht im Abstand von 50 sažen (ca. 100 Meter) aufgestellt werden sollten. In der Dunkelheit waren die Streckenposten angewiesen, Fackeln zu entzünden und so die

und Terror, S. 70; Naimark, Terrorism, S. 180 f. Seit April 1866 stand dem Kaiser eine persönliche Wachmannschaft (ochranitel'naja komanda) zur Verfügung, deren Aufgabe es war, den Herrscher während Staatsbesuchen zu beschützen. Vgl. Jonathan W. Daly, Autocracy under Siege. Security Police and Opposition in Russia, 1866-1905, Dekalb 1998, S. 19. Erst am 20.12.1883 wurde jedoch eine eigene Polizeieinheit (ochrannaja agentura) geschaffen, die speziell für die Sicherheit des Zaren und des Thronfolgers während deren Fahrten durch die Hauptstadt zuständig war. Vgl. Političeskaja policija i političeskij terrorizm v Rossii, S. $112 \mathrm{f}$.

56 Wortman, Scenarios of Power, S. 237.

57 Richard von Pfeil und Klein-Ellguth, Neun Jahre in russischen Diensten unter Kaiser Alexander III., Leipzig 1907, S. 247.

58 Zit. nach Wortman, Scenarios of Power, S. 432, Fn. 42.

59 Von Pfeil und Klein Ellguth, Neun Jahre in russischen Diensten unter Kaiser Alexander III., S. 247. 
Strecke des Hofzuges punktuell zu beleuchten. ${ }^{60}$ Neben der Aufstellung von Streckenposten mussten alle Einrichtungen der Bahnanlagen vor der Durchfahrt des Hofzuges sorgfältig kontrolliert werden. Stationsvorsteher wurden angewiesen, herumliegende Balken und Schwellen vom Bahngelände zu entfernen und sicher in Schuppen zu versperren. Baumaterial dieser Art, das entlang der Bahndämme auf offener Strecke lagerte, musste zum nächsten Streckenwärterhäuschen oder zur nächsten Kaserne gebracht werden. So wollte man verhindern, dass Übeltäter Gegenstände dieser Art auf die Gleise werfen konnten, um die Fahrt des Zarenzuges zu blockieren. Sicherheitsmaßnahmen dieser Art erinnern indes eher an die Zeit, als Staatsoberhäupter ihr Land noch mit der Kutsche bereisten. Dass Terroristen den Gleiskörper einer Bahnanlage untertunneln und verminen könnten, um so einen Unfall des Zarenzuges herbeizuführen, lag offenbar noch außerhalb des Vorstellungsvermögens der zarischen Sicherheitsbehörden. Als der Fürst Dmitrij D. Obolenskij, der den Anschlag vom 19. November 1879 als einer der Passagiere im Zug des kaiserlichen Gefolges überlebte, unmittelbar nach dem Unglück nach Moskau eilte, um dem Minister des Hofes, Graf Adlerberg, dem Chef der Dritten Abteilung, General-Adjutant Drentel'n, und dem GeneralGouverneur von Moskau, Fürst Dolgorukov, Bericht abzustatten, wollte keiner der drei hochrangigen Regierungsvertreter glauben, dass das Zugunglück durch einen terroristischen Bombenanschlag auf den Hofzug ausgelöst worden sein sollte. ${ }^{61}$ Graf Adlerberg soll der Hiobsbotschaft Obolenskijs entgegnet haben:

Wissen Sie, es kommt vor, dass die Nerven nach einem [Zug-]Unglück blank liegen. Ruhen Sie sich erst einmal aus; wenn Sie ausgeschlafen sind, sieht die Sache schon ganz anders aus. $^{62}$

Es stellte sich jedoch bald heraus, dass der Bericht Obolenskijs der Wahrheit entsprach. Die Tatsache, dass es Terroristen gewagt hatten, innerhalb der Stadtgrenzen von Moskau einen Bombenanschlag auf den Hofzug Alexanders II.

60 Vgl. Zirkular des Chefingenieurs der Nikolaj-Bahn an die Verantwortlichen für die Sicherung der Strecke anlässlich der Reise Alexanders II. im November 1879. Central'nyj Gosudarstvennyj Istoričeskij Archiv Sankt Peterburga (im folgenden CGIA SPB) f. 1480, op. 17, ed. chr. 1, 1. 171. Ein sažen entspricht 2,13 Meter.

61 Nabroskij vospominanij knjazja D. D. Obolenskogo, S. $272 \mathrm{f}$.

62 Ebd., S. 272. Dass die Möglichkeit eines Bombenanschlags auf die Bahnstrecke des Zarenzuges im November 1879 noch außerhalb der Vorstellungsmöglichkeiten der zarischen Sicherheitsbehörden lag, belegt auch die Tatsache, dass die Festnahme eines Aktivisten der Gruppe Narodnaja volja in Elizavetgrad, unmittelbar vor dem Anschlag bei Moskau nicht zu einer Verschärfung der Sicherheitsmaßnahmen entlang der Bahnlinie führte, obwohl man bei Goldenberg große Mengen Dynamits fand. Vgl. dazu: Figner, Polnoe Sobranie sočinenij, Bd. 1, S. 187; Meščerskij, Vospominanija, S. 440; Rutkowski, Revolutionäre Bewegung, S. 395. 
zu unternehmen, wirkte auf die zarische Elite wie ein Schock - und zugleich elektrisierend. Dementsprechend lässt sich nach dem Attentat von 1879 eine deutliche Veränderung des Sicherheitsbewusstseins innerhalb der Bürokratie beobachten. Von nun an zogen es nervöse Streckenwärter der Bahnanlagen z. B. vor, den Hofzug des Zaren auf offenem Feld anzuhalten, um ja nicht das Risiko einzugehen, möglicherweise ein verdächtiges Erdloch in der Nähe der Gleise ignoriert $\mathrm{zu}$ haben. Als ein Soldat, der mit der Bewachung einer Bahnstrecke in der Nähe der Stadt Fastov in den 1880er Jahren betraut war, den Zug Alexanders III. auf offenem Feld anhielt, weil er ein Kartoffellager in der Nähe der Bahnanlage für das Versteck einer Bombe hielt, wurde er vom mitreisenden Kriegsminister für seine Wachsamkeit ausdrücklich gelobt. ${ }^{63}$ Die Gefahr terroristischer Anschläge wurde auch in den überarbeiteten „Instruktionen für die Bewachung des Kaiserlichen Zuges während der Reisen hochgestellter Persönlichkeiten" explizit erwähnt. ${ }^{64}$ In den Richtlinien aus dem Jahr 1891 wurden die Streckenwärter beispielsweise angewiesen, alle notwendigen Maßnahmen zu ergreifen, um einen (terroristischen) Anschlag auf den fahrenden Hofzug zu vereiteln. Insbesondere galt es, einen außerplanmäßigen Halt oder einen Unfall des Zuges durch mutwillige Beschädigungen von Brücken und Gleisen, die Untertunnelung des Bahnkörpers oder durch einen vergrabenen Sprengsatz zu verhindern. Die Sicherheitskräfte hatten ihre Lehre aus den Anschlägen im November 1879 gezogen und wiesen die Polizei nun an, insbesondere auf Personen zu achten, die in der Nähe von Bahnanlagen wohnten oder die gerade erst dorthin gezogen waren $(\$ 14)$. Vor der Durchfahrt des Zarenzuges mussten alle Bahnanlagen und Brücken aufs Genaueste untersucht und überprüft werden. Besondere Aufmerksamkeit sollten die Verantwortlichen dabei Gebäuden in der Nähe des Bahndammes und den Überresten frischer Erdarbeiten schenken ( $\$ \$ 18$ u. 19). ${ }^{65}$ Während der Fahrt des Hofzuges musste die Strecke nun tagsüber in einem Abstand von 50 sažen (des Nachts 25 sažen) mit Wachen besetzt werden (\$25). Die zuletzt

63 Stranička iz prošlago, in: Istoričeskij vestnik 33. 1912, S. 508 - 512.

64 Instrukcija po ochrane imperatorskich železnodorožnych poezdov pri vysočajšich putešestvijach, Sankt Peterburg 1891; CGIA SPB f. 1374, op. 1, ed. chr. 344, 1. 17 - 30.

65 Appell im Zirkular vom Korpus Žandarmov, Nr. 155 vom 7.11.1906, an die Leiter der einzelnen upravlenija, insbesondere auf frische Erdarbeiten an den Strecken zu achten, da diese ggf. auf einen geplanten Bombenanschlag hindeuten können. Abdruck bei: Lev Timofeevič, Spravočnaja kniga dlja činov Žandarmskich Policejskich Upravlenij železnych dorog po žandarmsko-policejskoj časti. Obščija objazanosti žandarmskoj železnodorožnoj policii, Sankt Peterburg 1908, S. 13. Dass diese Instruktionen im Bewusstsein des Wachpersonals verinnerlicht worden waren, verdeutlicht der Bericht von Richard von Pfeil und Klein-Ellguth, Neun Jahre in russischen Diensten unter Kaiser Alexander III., S. 248, in dem der Autor beschreibt, man habe bei der Kontrolle der Bahnanlagen insbesondere auf „neuerdings angelegte Erdarbeiten, Brücken und die unter dem Bahndamme führenden Röhren“ geachtet. 
erwähnte Sicherheitsmaßnahme bedeutete in der Praxis, dass man zu Beginn der 1890er Jahre bereits doppelt so viel Personal für die Bewachung der Strecken einer kaiserlichen Zugreise benötigte als Ende der 1870er Jahre. ${ }^{66}$ Dies führte nicht zuletzt zu einer Explosion der Kosten der entsprechenden Sicherheitsmaßnahmen. Die Bewachung der Eisenbahnstrecke von St. Petersburg an die Westgrenze des Reiches, die Alexander III. mit seiner Familie im Herbst 1888 auf der Fahrt nach Dänemark befuhr, sollen für die Hin- und Rückreise die astronomische Summe von 750.000 Rubel gekostet haben. ${ }^{67}$ In den folgenden Jahren wurden auch diese Sicherheitsbestimmungen weiter verschärft. In diesem Zusammenhang geriet zunehmend auch die Infrastruktur von Telefon und Telegraf ins Blickfeld der zarischen Sicherheitsbehörden. ${ }^{6} 1892$ wurden beispielsweise Bahnhofsvorsteher angewiesen, eine Stunde vor der fahrplanmäßigen Ankunft bzw. Abfahrt des kaiserlichen Zuges keine privaten Telegramme mehr anzunehmen bzw. weiterzuleiten. Zudem mussten zu dieser Zeit auch alle Räumlichkeiten, in denen sich ein Telefon befand, sicher verschlossen werden. ${ }^{69}$ Zwei Jahre später wurden die Mitarbeiter der Warschau-Petersburg-Bahn in einem Rundschreiben angewiesen, dass der Zug des Zaren in Telegrammen nicht mehr „kaiserlicher Zug“, sondern nur noch „Sonderzug“ (ékstrennyj poezd) genannt werden dürfe. ${ }^{70}$ Aus Angst vor seiner eigenen Bevölkerung und vor der Gefahr politisch motivierter Gewalt hatte der Zar begonnen, seine Reisen im eigenen Land auch in Telegrammen zu tarnen.

Als sich Zar Nikolaus II. im Jahr 1912 auf die Reise nach Borodino machte, um dort der Hundertjahrfeier der Schlacht gegen Napoleon beizuwohnen, wurden nicht nur die Brücken und die anderen strategischen Punkte der Bahnstrecke

66 Listen, die Auskunft über Anzahl und Namen der für die Bewachung der Reise des Zaren auf der Nikolaj-Bahn im November 1878 angeheuerten Personen geben, finden sich in: CGIA SPB f. 1480, op. 17, ed. chr. 1, 1l. 13 - 120.

67 Vgl. von Pfeil und Klein-Ellguth, Neun Jahre in russischen Diensten unter Kaiser Alexander III., S. 249. Von Pfeil und Klein-Ellguth war Kapitän, ab 1888 Bataillonskommandant im Preobraženskij-Garderegiment des Kaisers und im November 1888 mit der Bewachung eines 300 Kilometer langen Streckenabschnitts zwischen St. Petersburg und Pskov betraut.

68 Die Aktivisten der Narodnaja volja hatten bereits im November 1879 den Eisenbahntelegrafen zur Kommunikation zwischen Simferopol und Moskau genutzt. Vgl. Nabroskij vospominanij knjazja D. D. Obolenskogo, S. 271.

69 Instrukcija načal'nikam stancii i ich zamestitel'jam pri sledovanii imperatorskich poezdov, hg.v. Glavnoe obščestvo Rossijskich železnych dorog. Linija S. PeterburgoVaršavskaja, Sankt Petersburg 1892, \$\$ 27, 33; CGIA SPB f. 1374, op. 1, ed. chr. 346, 11. 6-20. Dieses Dokument basiert auf der Položenie ob imperatorskich poezdach, hg.v. Ministerstvo Putej Soobščenija, 24.4.1892, Sankt Petersburg 1892, § 86-96.

70 CGIA SPB f. 1374, op. 1, ed. chr. 344, 1. 11. 
streng bewacht. ${ }^{71}$ Zudem mussten sich alle Menschen, die entlang der Strecke bzw. in den Städten wohnten, die der Kaiser besuchen wollte, im Vorfeld polizeilich registrieren lassen. So sollte verhindert werden, dass sich verdächtige Personen der Reiseroute des Kaisers nähern können. ${ }^{72}$ Sicherheitsmaßnahmen dieser Art trugen dazu bei, dass sich die Regenten, deren Mobilität innerhalb ihres Landes durch die Modernisierung der Infrastruktur theoretisch immer mehr zunahm, in Wirklichkeit immer stärker von ihrer eigenen Bevölkerung entfernten. Dies führte im Falle Alexanders III. dazu, dass sich der Zar zunehmend in seinem festungsartigen Schloss im Petersburger Vorort Gatčina verschanzte und - im Gegensatz zu seinem Vater - sein eigenes Land nur äußerst selten bereiste. ${ }^{73}$

Diese zunehmende Abschirmung des Zaren von der eigenen Bevölkerung wurde von den Aktivisten der Narodnaja volja mit großer Genugtuung registriert und als Beweis für die eigene Macht im Kampf mit der Autokratie interpretiert. Als Alexander II. zwei Tage nach dem misslungenen Anschlag auf seine Person am 19. November 1879 (angeblich) den ca. 30 Meter weiten Weg von der Uspenskij-Kathedrale zum Cudov-Kloster im Moskauer Kreml' nicht zu Fuß, sondern in einer von Kosaken eskortierten Kutsche zurücklegte, jubelte die Untergrundzeitung von Narodnaja volja: „das Publikum sah nichts als einen großen Konvoi, in der Menge sagte man, dass sie den Zaren wie einen Gefangenen unter der Bewachung von Tscherkessen abführen. ${ }^{\text {"74 }}$ In einem fiktiven Tagebuch Alexanders II., das am 20. September 1880 in der illegalen Zeitung Listok Narodnoj voli verbreitet wurde, lassen die Autoren den Selbstherrscher resignierend notieren:

Früher konnte man noch in der offenen Kutsche fahren und mit [seinen] zwei Hunden spazieren gehen ... Und heute? Ein ordentlicher Mensch darf heute nicht mehr auf die Straße gehen! Es ist heiß und schwül - ich klettere in meine Kutsche und verbarrikadiere mich darin [zakuporivajsja]; nur noch im Schatten des Tscherkessen, der mit seinem Körper das Kutschenfenster verdeckt, kann man sich vor der Glut der Sonne verstecken. Ich muss nur ein paar sažen zurücklegen, aber ich bin gezwungen, kreuz und quer zu fahren und ,Haken zu schlagen wie ein Hase. Wie schwierig ist es geworden, den Quell der Wohltaten Russlands zu beschützen! $!^{75}$

71 GARF f. 77, op. 1, ed. chr. 95, 1. $261-263$.

72 Ebd., 1. 170.

73 Wortman, Scenarios, S. 263. Demgegenüber betont der Gardeoberst von Pfeil und KleinEllguth, dass es leicht gewesen sei, den Kaiser in Peterhof zu sehen, „wenn auch der Nihilisten wegen einige Absperrungsmaßregeln geboten waren“. Ders. Neun Jahre in russischen Diensten unter Kaiser Alexander III., S. 77.

74 Narodnaja volja. Social'no-revoljucionnoe obozrenie, 1.1.1880, S. 14, in: Literatura partii „Narodnaja volja“, S. 58.

75 Listok Narodnoj voli. Revoljucionnaja chronika, 20.9.1880, S. 7 -8, in: Literatura partii „Narodnaja volja“, S. 89-90. Vgl. auch die Glosse Vot tebe deduška, i jur'ev den’ in der in 
Einigen Zeitgenossen war durchaus bewusst, dass die immer umfassenderen Sicherheitsvorkehrungen, die die Behörden seit 1879 zum Schutz des Monarchen trafen, im Grunde genommen nur symbolische Bedeutung hatten. Der im Herbst 1888 mit der Bewachung eines $150 \mathrm{~km}$ langen Streckenabschnitts der Bahnlinie von St. Petersburg nach Pskov betraute Gardeoberst Richard von Pfeil und Klein Ellguth bezeichnete den Nutzen des Bewachungsdienstes der vom Zaren genutzten Eisenbahngleise als „Zweifelhaft“: „Wollte wirklich ein entschlossener Übeltäter versuchen, den Zug zum Entgleisen zu bringen, so konnten ihn, namentlich in finsteren Nächten, die Posten kaum entdecken. ${ }^{\text {"76 }}$ Schon wenige Tage nach dem Sprengstoffanschlag im Winterpalast im Februar 1880 hatte der österreichische Botschafter in St. Petersburg Graf Gustav Kálnoky nach Wien telegrafiert:

Kein Mensch [am russischen Hof] hat eine Ahnung, wie die Frage der Sicherheit des Kaisers gelöst werden kann; man ist ganz ratlos hierin, gar nicht zu sprechen von der entsetzlichen inneren Situation überhaupt. ${ }^{77}$

Es wirkt wie eine Ironie des Schicksals, dass das legendäre Zugunglück am 18. Oktober 1888 bei Borki, bei dem der kaiserliche Zug Alexanders III. völlig zerstört wurde, das der Zar und seine Familie aber wie durch ein Wunder unverletzt überlebten, nicht die Folge eines terroristischen Anschlags, sondern des Geschwindigkeitsrausches des Zaren und des schlechten Zustands der Bahnanlagen bei Char'kov war. ${ }^{78}$ Der Unfall des Hofzuges setzte dem einzigen ceremonial trip Alexanders III., der ihn in die Ukraine, nach Polen und in den südlichen Kaukasus führte, ein jähes Ende. Nach dem Unfall, der 22 Todesopfer unter dem kaiserlichen Gefolge forderte, unternahmen die Behörden jede Anstrengung um zu betonen, dass der kaiserliche Zug nicht einem Terroranschlag zum Opfer gefallen war. In dem offiziellen Communiqué, das der Hofminister schon am folgenden Tag verbreiten ließ, hieß es, dass „nichts auf einen kriminellen Hintergrund“ dieses Vorfalls hindeute (o kakom-libo zloumyšlenii $v$ etom nesčastnom

Genf erschienen revolutionären Zeitung Obščee delo, 1879, Nr. 29, S. 1 f. Für den Hinweis auf diese beiden Quellen danken wir Julija Safronova.

76 Von Pfeil und Klein Ellguth, Neun Jahre in russischen Diensten unter Kaiser Alexander III., S. 249.

77 Zit. nach Rutkowski, Revolutionäre Bewegung, S. 410. Die Ohnmacht der Behörden, die Sicherheit des Zaren sowohl im öffentlichen Raum als auch in den privaten Gemächern $\mathrm{zu}$ gewährleisten, beschreibt auch der einflussreiche Publizist Vladimir Petrovič Meščerskij in seinen Memoiren. Ders., Moi vospominanija. Čast vtoraja, S. 441.

78 Zum Zugunglück von Borki vgl. u. a. Wortman, Scenarios, S. 310-311; Anatolij Fedorovič Koni, Krušenie carskogo poezda v 1888 godu (Borki-Taranovka), in: ders., Sobranie sočinenija v 8-mi tomach, Bd. 1, Moskau 1966, S. 420-495; Von Pfeil und KleinEllguth, Neun Jahre in russischen Diensten unter Kaiser Alexander III., S. 240-246; Sergej Jul'evič Vitte, Izbrannye vospominanija 1849-1911, Moskau 1991, S. 121 - 135. 
slučae ne možet byt' i reči). ${ }^{79}$ Selbst der Satz, dass der Unfall „nicht von einem Missetäter geplant worden sei“ (vytekajut ne iz umysla prestupnago) [unsere Hervorhebung], der sich noch im Entwurf des offiziellen Reskripts zu dem Unfall findet, wurde in der am 15. Mai 1889 im Pravitel'stvennyj vestnik veröffentlichten Fassung des Dokuments gestrichen. ${ }^{80}$ Aus der Sicht der Behörden erschien selbst die Idee, dass das Zugunglück einen terroristischen Hintergrund gehabt haben könnte, zu gefährlich zu denken.

Dank des erbitterten Widerstandes aus dem russischen Verkehrsministerium wurde die russische Öffentlichkeit über die eigentlichen Gründe und die Verantwortlichen des Unfalls von 1888 im Unklaren gelassen. Für diese Fragen schien sich jedoch ohnehin kaum jemand zu interessieren, denn noch wichtiger als die Frage, wie es zu dem Unglück bei Borki kam, war, wie der Kaiser und seine Familie diesem Eisenbahninferno unverletzt entkommen konnten. Schon im offiziellen Communiqué des Hofministers vom 18. Oktober hieß es:

[Es ist] unmöglich zu glauben, dass irgendjemand einem solchen Unfall unbeschadet entkommen kann. Aber Gott schützte den Zaren und seine Familie: Sie entstiegen den zertrümmerten Waggons, ohne Wunden davonzutragen. ${ }^{81}$

Die Rettung der kaiserlichen Familie wurde erneut als „Wunder“ und als Ausdruck göttlicher Gnade propagiert und von vielen Menschen in dieser Form wahrgenommen. In seinen Erinnerungen hielt der deutsche Botschafter General von Schweinitz fest, „seit dem, Wunder von Borki‘ glaubt man [in der Petersburger Gesellschaft], dass niemand mehr es wagen würde, das so sichtbar von der Vorsehung geschützte Leben des Zaren zu bedrohen. " ${ }^{82}$ Wie nach dem Attentatsversuch von 1879 kam auch nach dem „Wunder von Borki“ in Russland ein kolorierter Volksbilderbogen (lubok) in Umlauf, der zeigte, wie ein Engel seine schirmende Hand über den Waggon des zertrümmerten Zuges hielt, in dem die kaiserliche Familie am 18. Oktober 1888 gereist war. ${ }^{83}$ Auch die Errichtung einer Gedenkkapelle am Ort der wundersamen Rettung

79 Offizielles Telegram des Ministers des Kaiserlichen Hofes vom 18.10.1888. Zit. nach Nedelja. Eženedel'naja gazeta, Nr. 43, 23.10.1888, S. 1351 - 1354. Zum Unfall von Borki vgl. auch: Rossijskij Gosudarstvennyj Istoričeskij Archiv (im folgenden RGIA) f. 1224, op. 1 , ed. chr. 1.

80 RGIAf. 1224, op. 1, ed. chr. 1, 1. 153. Zur erbitterten Diskussion zwischen den verschiedenen Behörden der Reichsregierung um den Text des Reskripts vgl. Koni, Sobranie sočinenij, Bd. 1, S. $487-491$.

81 Offizielles Telegramm des Ministers des Kaiserlichen Hofes vom 18. 10.1888.

82 Denkwürdigkeiten des Botschafters Generals von Schweinitz, Bd. 2, S. 386.

83 Lubok aus dem Jahr 1888, genehmigt von der Zensur am 16.11.1888. Chromo-Lithografie, M. S. Solov'ev, Moskau. Titel am unteren Bildrand: „Providenie Božie Spasaet Gosudarja i Ego Semejstvo“. Russische Nationalbibliothek St. Petersburg. Abteilung für Drucke, Signatur: Ė 1-ir-485,1 / 7-3. 
Alexanders II. am 4. April 1866 vor den Schüssen Karakosovs, fand ihre Entsprechung im Bau eines gewaltigen Gotteshauses, das der Zar nach dem Zugunglück von Borki am Ort des Geschehens errichten ließ. ${ }^{84}$ „Für alle Zeit“ wurden am Erinnerungstage Dankgottesdienste in Russland festgesetzt. ${ }^{85}$

Hier zeigt sich, dass nicht nur die Verhaltensmuster der russischen Monarchen an vormodernen Strukturen soldatisch-aristokratischer Tugendhaftigkeit orientiert blieben. Auch in der Außendarstellung und in der Wahrnehmung eines großen Teils der Öffentlichkeit blieb die Frage der Sicherheit und des Schutzes des gesalbten Autokraten letztendlich eine Angelegenheit, die außerhalb der Reichweite menschlichen Handelns lag. Sowohl die Wachen Alexanders II. die ihn nach der ersten Detonation am 1. März 1881 drängten, mit dem Schlitten so schnell wie möglich den Unglücksort zu verlassen, als auch die Beamten, die immer strengere Reglements für die Bewachung des Zaren auf seinen Reisen entwarfen, hatten bereits erkannt, dass sich die Frage der Sicherheit eines Regenten im ausgehenden 19. Jahrhunderts anders darstellte als noch wenige Jahrzehnte zuvor. Diese Erkenntnis konnte jedoch weder das Verhalten der Regenten, noch die gesellschaftlichen Perzeptionsmuster von Gefahrensituationen kurzfristig verändern. Es war allerdings nur eine Frage der Zeit, bis sich auch in Russland die Erkenntnis durchsetzte, dass der Körper des Königs im Zeitalter der Dampfmaschine und des Dynamits ganz neuen Gefährdungen ausgesetzt ist, die auch ein neues handlungsleitendes Sicherheitsbewusstsein erforderten.

\section{Epilog: George W. Bush am 11. September 2001}

„A second plane hit the second tower. America is under attack." Mit diesen Worten informierte White House Chief of Staff Andrew Card am 11. September 2001 um 9.05 Uhr Präsident George W. Bush leise über die Angriffe auf das World Trade Center in New York. ${ }^{86}$ Bush befand sich über 2.000 Kilometer

84 Vgl. Richard Wortman, The „Russian Style“ in Church Architecture as Imperial Symbol after 1881, in: James Craraft u. Daniel Rowland (Hg.), Architectures of Russian Identity. 1500 to the Present, Ithaca 2003, S. $101-116$, bes. S. $108-110$.

85 Von Pfeil und Klein-Ellguth, Neun Jahre in russischen Diensten unter Kaiser Alexander III., S. 241. Der 17. Oktober blieb auch für Nikolaus II., der als Großfürst den Unfall überlebte, ein wichtiges Datum der Erinnerung. Dies wird aus den Eintragungen in seinem Tagebuch deutlich. Vgl. Dnevniki Imperatora Nikolaja II. hg.v. Kornelij Fedorovič Šacillo, Moskau 1991, S. 42 (1894), S. 175 (1896), S. 234 (1904), S. 285 (1905), S. 338 (1906), S. 416 (1913), S. 492 (1914).

86 Für diese und die weiteren Zeit- und Ortsangaben vgl. National Commission on Terrorist Attacks Upon the United States, The 9/11 Commission Report, New York 2004, S. 38 ff. Die Abläufe dieses Tages sind ein Politikum. Vgl. einerseits Bill Sammon, Fighting Back. The War on Terrorism. From Inside the Bush White House, Washington 
von den Angriffen entfernt in einer Grundschule in Florida. Er blieb für etwa weitere fünf Minuten in dem Klassenzimmer, bis er in einen Nebenraum ging, dort die Fernsehbilder sah und von seinen Mitarbeitern auf den neusten Stand der Ereignisse gebracht wurde. Er entschied sich dafür, von der Schule aus ein kurzes Statement abzugeben und dann zum Flughafen zurückzukehren. Eine halbe Stunde nachdem er erstmals informiert worden war, verließ die Autokolonne das Schulgelände. Dem Bericht der National Commission zufolge,

the Secret Service agent felt strongly that the situation in Washington was too unstable for the President to return there $[\ldots]$ The President strongly wanted to return to Washington and only grudgingly agreed to go elsewhere.

Vize-Präsident Richard B. Cheney, der selbst sofort in einen Bunker evakuiert worden war, riet Bush von einer Rückkehr nach Washington ab. Als Air Force One abhob, stand eine Entscheidung noch aus: „The objective was to get up in the air - as fast and as high as possible - and then decide where to go. ${ }^{\text {“87 }}$ Erst in der Luft fiel die Entscheidung, Bush zu der Bardsdale Air Force Base in Louisiana zu fliegen, wo der Präsident eine weitere kurze Ansprache hielt; von dort aus ging es weiter zum US Strategic Command Center in Nebraska, dessen Bunker alle technischen Voraussetzungen für eine National Security Conference bot. Dabei war der amerikanischen Öffentlichkeit der Aufenthaltsort ihres Präsidenten vom Abflug aus Florida bis zur Landung in Nebraska vollkommen unbekannt. Nach der Sitzung der National Security Conference flog Bush nach Washington und kam abends um kurz vor sieben Uhr im Weißen Haus an. Dort gab er anderthalb Stunden später eine weitere fünfminütige Fernsehansprache.

Zwischen den Attentaten auf König Wilhelm I., Queen Victoria sowie Zar Alexander II. und den Anschlägen auf das World Trade Center in New York und das Pentagon in Washington liegen gut anderthalb Jahrhunderte. In vieler Hinsicht sind diese terroristischen Gewalttaten so verschieden, dass ein Vergleich nur schwer möglich erscheint. So ist bis heute nicht klar, ob Al-Qaida den Präsidenten der Vereinigten Staaten treffen wollte. Das vierte Flugzeug, United 93, das durch die Intervention der Passagiere in Pennsylvania abstürzte, sollte vielleicht das Weiße Haus, womöglich aber auch das Capitol zerstören. ${ }^{88}$

2002 und andererseits etwa Allan Wood u. Paul Thompson, An Interesting Day. President Bush's Movements and Actions on 9/11, unter: Center for Cooperative Research/ History Commons. A Project of the Center for Grassroots Oversight, http://www.cooperativeresearch.org/essay.jsp?article=essayaninterestingday. Bei sich widersprechenden Angaben folgt diese Darstellung der Rekonstruktion der National Commission.

87 Beide Zitate in National Commission on Terrorist Attacks Upon the United States, The 9/ 11 Commission Report, S. 39. Vgl. auch die ausführliche Darstellung dieser Entscheidung in Sammon, Fighting Back, S. $100-135$.

88 Vgl. National Commission on Terrorist Attacks Upon the United States, The 9/11 
Hier kommt es jedoch nur auf einen Punkt an: die Reaktion des Präsidenten und seiner Mitarbeiter. Auch wenn Bush seiner Aussage nach so schnell wie möglich nach Washington zurückkehren wollte, setzte sich der Secret Service durch. Wie Bush später im Interview erläuterte, gaben bei dieser Entscheidung Überlegungen formaler Art den Ausschlag:

When the president is under threat, one thing for the good of the country is you want to remove the president from the immediate threat. There's nothing worse for a country having been attacked than a destabilized presidency. It would make matters a lot worse. The best thing to do is exercise caution and to get the president to a position where he can be in communications with his team. And you don't have to be looking at each other to be in communication. You just have got to be in communication. ${ }^{89}$

In Bushs Person musste der Präsident der Vereinigten Staaten von Amerika als Institution geschützt werden - um seiner selbst willen -, aber vor allem um die politische Handlungsfähigkeit der USA zu erhalten. Und dies konnte am besten durch Rückzug und Ausweichen in den Luftraum und auf abgelegene Militärbasen gewährleistet werden, bis die Situation besser einschätzbar sein würde. Auf einen modernen, hochtechnisierten und entpersonalisierten Anschlag erfolgte eine moderne, entpersonalisierte Sicherheitsantwort. Das Modell soldatisch-aristokratischer Tugendhaftigkeit hatte ausgedient - scheinbar zumindest, denn genau diese vorsichtige Reaktion wurde in der Öffentlichkeit später scharf angegriffen.

Bush „skedaddled“ - er nahm Reißaus, verdünnisierte sich - so ein Kolumnist im Daily Courier aus Oregon: „The airline passengers who struggled with hijackers before crashing in Pennsylvania are the heroes“, heißt es weiter: „Against their courage the picture of Bush hiding in a Nebraska hole becomes an embarrassment." Und Tom Gutting warf dem Präsidenten unter der Überschrift „Bush has failed to lead the U.S.“ in der Texas City Sun vor, he was "flying around like a scared child, seeking refuge in his mother's bed after having a nightmare." Beide Journalisten wurden direkt nach diesen Kommentaren entlassen. Daraufhin griffen die Washington Post, die New York Times sowie Internetforen das Thema auf, womit es den Charakter von national news bekam und breit diskutiert wurde. ${ }^{90}$ Präsenz am Unglücksort, Mut zur Konfrontation und Unerschrockenheit, die sich im Notfall bis zur Lebensverachtung steigert, sind folglich nach wie vor Eigenschaften, die im ge-

Commission Report, S. 10 - 14. Die Warnung, dass Air Force One, das nächste Ziel nach dem Pentagon sein würde, beruhte auf Fehlinformationen.

89 Sammon, Fighting Back, S. 130 u. S. 119.

90 Siehe etwa Bill Carter u. Felicity Barringer, In Patriotic Time, Dissent is Muted, in: The New York Times, 28.9.2001; Richard Reeves, Patriotism Calls Out the Censor, in: The New York Times, 1.10.2001; Howard Kurtz, What Bush Said And When He Said It, in: The Washington Post, 1.10.2001 oder im Internet Joel Davis, Columnists Fired After Criticizing Bush, in: Free Republic, 28.9.2001, http://www.freerepublic.com/focus/ f-news/534757/posts. 
sellschaftlichen Erwartungshorizont gegenüber Staatsoberhäuptern liegen. Wie dies mit der Logik moderner, formalisierter und technisierter Sicherheitserwägungen in Übereinstimmung zu bringen ist, bleibt vorerst jedoch eine offene Frage.

Dr. Carola Dietze, Deutsches Historisches Institut Washington, 1607 New Hampshire Ave. N.W., Washington, D.C. 20009, USA

E-Mail: dietze@ghi-dc.org

Dr. Frithjof Benjamin Schenk, Ludwig-Maximilians-Universität München, Historisches Seminar, Geschwister-Scholl-Platz 1, D-80539 München

E-Mail: B.Schenk@lmu.de 